\title{
Indolylthio glycosides as effective building blocks for chemical glycosylation
}

Ganesh Shrestha, Matteo Panza, Yashapal Singh, Nigam P. Rath, and Alexei V. Demchenko*

Department of Chemistry and Biochemistry, University of Missouri - St. Louis, One University Boulevard, St. Louis, Missouri 63121, USA

Email: demchenkoa@umsl.edu

\section{$\underline{\text { Contents }}$}

Additional ${ }^{1} \mathrm{H}$ NMR monitored experiments $\quad$ S1

$\begin{array}{ll}\text { NMR Spectra of new compounds } & \text { S7 }\end{array}$

NMR spectra of recovered products from competition experiments $\quad$ S16

$\begin{array}{ll}\text { NMR spectra of known disaccharides } & \text { S18 }\end{array}$

$\begin{array}{ll}\text { X-Ray crystal structure determination for compound } 2 & \text { S30 }\end{array}$

$\begin{array}{ll}\text { X-Ray crystal structure determination for compound } 6 & \text { S32 }\end{array}$

\section{$\underline{\text { Additional }}{ }^{1}$ H NMR monitored experiments}

\section{Deuterium exchange experiment}

A mixture of donor $2(30 \mathrm{mg}, 0.041 \mathrm{mmol})$ and molecular sieves $(80 \mathrm{mg})$ in 1,2-DCE $(1.0 \mathrm{~mL})$ was stirred for $1 \mathrm{~h}$ under argon at room temperature. After that, $N$-iodosuccinimide $(8.9 \mathrm{mg}, 0.041$ mmol) was added and stirring was continued for $17 \mathrm{~h}$. TLC was checked at intervals of 5 minutes, 1, 2 and $5 \mathrm{~h}$, no noticeable change was seen. After that, he reaction mixture was filtered through a pad of Celite, diluted with DCM $(30 \mathrm{~mL})$, washed with $10 \%$ aq. $\mathrm{Na}_{2} \mathrm{~S}_{2} \mathrm{O}_{3} .5 \mathrm{H}_{2} 0 .(10 \mathrm{~mL})$, and water $(2 \times 10 \mathrm{~mL})$. The organic phase was separated dried over sodium sulfate, filtered, and concentrated in vacuo. The residue was purified by column chromatography on silica gel (ethyl acetate/hexane gradient elution). ${ }^{1} \mathrm{H}$ NMR showed that the compound corresponds to 23 (Figure S1, Spectrum B). Mass spectroscopy also proved the compound corresponds to 23 (HR-FAB MS [M+Na] ${ }^{+}$: calcd for $\left[\mathrm{C}_{42} \mathrm{H}_{32} \mathrm{INO}{ }_{9} \mathrm{~S}+\mathrm{Na}\right]^{+}$876.0842; found: 876.0734 .

Deuterium exchange experiment was conducted by adding $4.0 \mu \mathrm{L}$ of $\mathrm{D}_{2} \mathrm{O}$ to a sample of the anticipated compound 23 in $\mathrm{CDCl}_{3}$, the ${ }^{1} \mathrm{H}-\mathrm{NMR}$ spectra again supported that the isolated compound to be 23 (Figure S1, Spectrum C) where disappearance of the N-H signal at $9.69 \mathrm{ppm}$ was observed. The data acquired from ${ }^{1} \mathrm{H}$ NMR spectroscopy and mass spectrometry confirmed the formation of compound $\mathbf{2 3}$. 

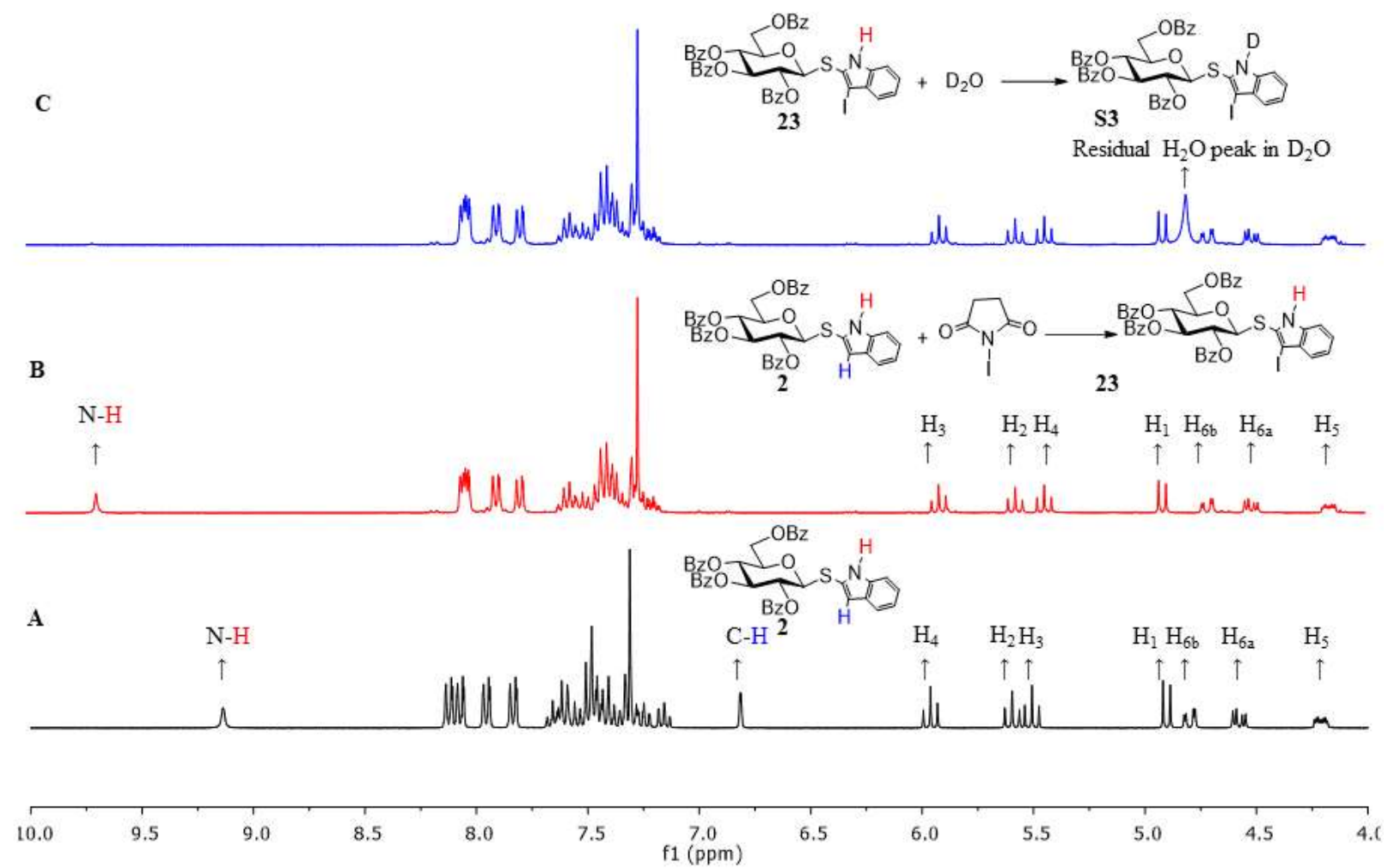

Figure S1: Deuterium exchange experiment

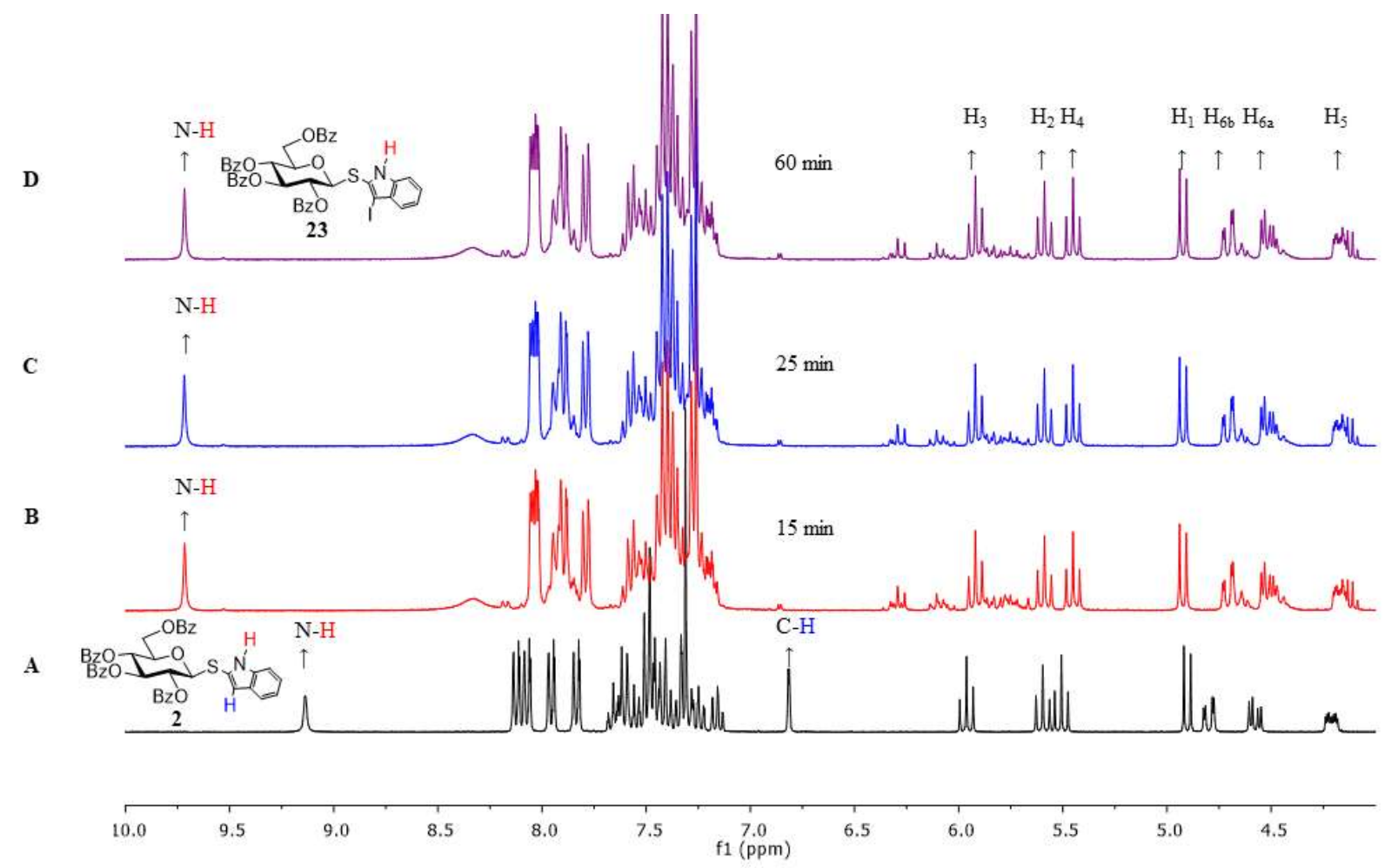

Figure S2: ${ }^{1} \mathrm{H}$ NMR spectra of A) glycosyl donor $\mathbf{2}$ in $\mathrm{CDCl}_{3}$; B) glycosyl donor $\mathbf{2}$ in $\mathrm{CDCl}_{3}$ in presence of 1 equiv. NIS at intervals of $15 \mathrm{~min}$; C) $25 \mathrm{~min}$; and D) $1 \mathrm{~h}$. 


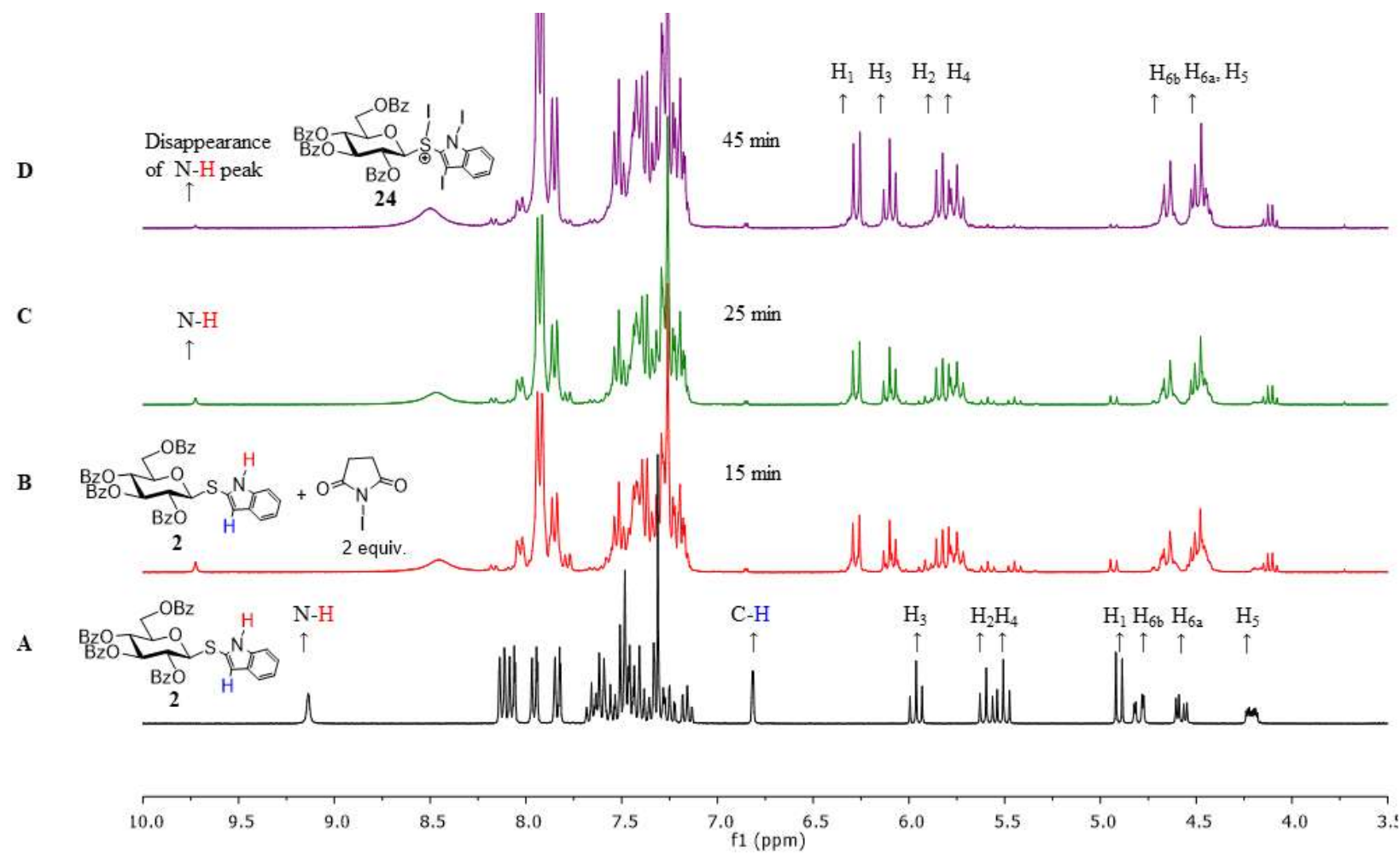

Figure S3: ${ }^{1} \mathrm{H}$ NMR spectra of A) glycosyl donor $\mathbf{2}$ in $\mathrm{CDCl}_{3}$; B) glycosyl donor $\mathbf{2}$ in $\mathrm{CDCl}_{3}$ in presence of 2 equiv NIS at intervals of $15 \mathrm{~min}$; C) in $25 \mathrm{~min}$; D) in $45 \mathrm{~min}$.

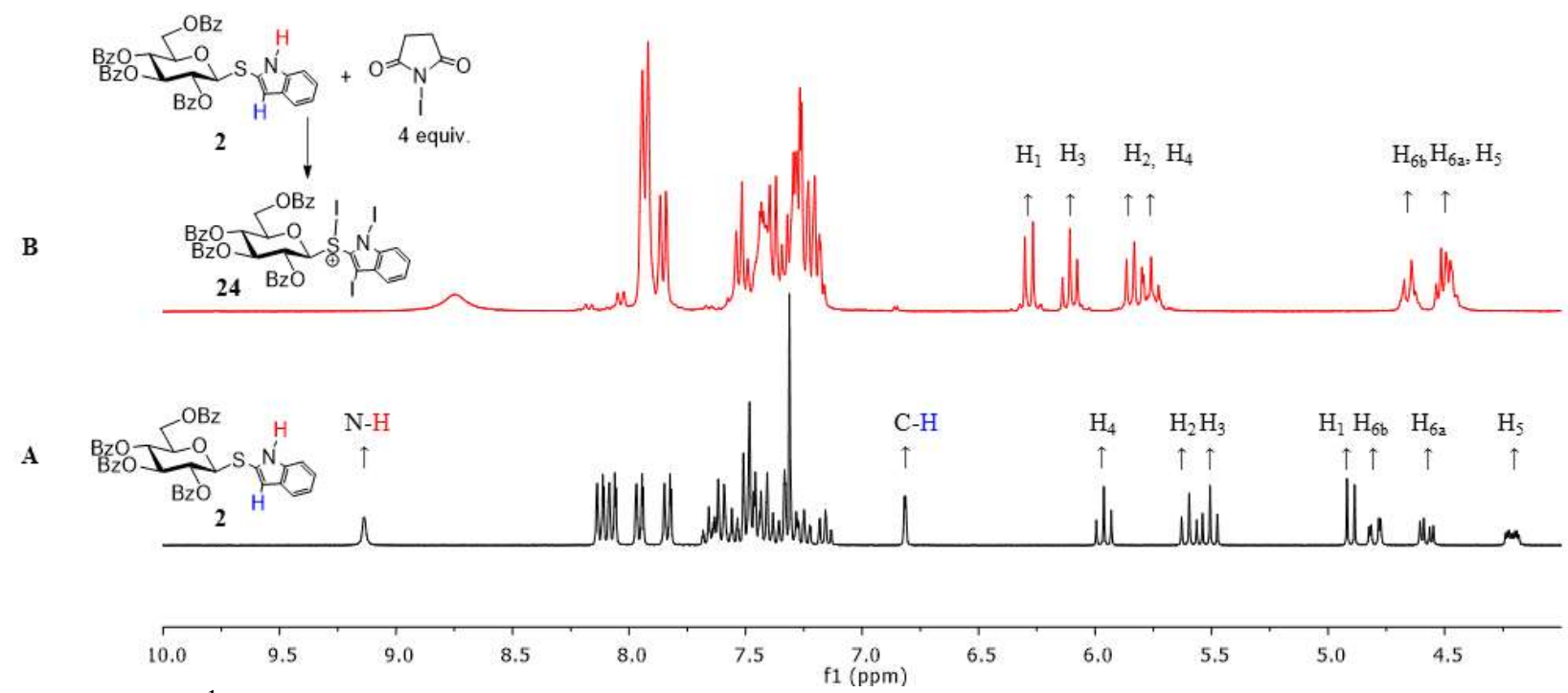

Figure S4: ${ }^{1} \mathrm{H}$ NMR spectra of A) glycosyl donor 2 in $\mathrm{CDCl}_{3}$; B) glycosyl donor $\mathbf{2}$ in $\mathrm{CDCl}_{3}$ in presence of 4 equiv of NIS after $1 \mathrm{~h}$. 

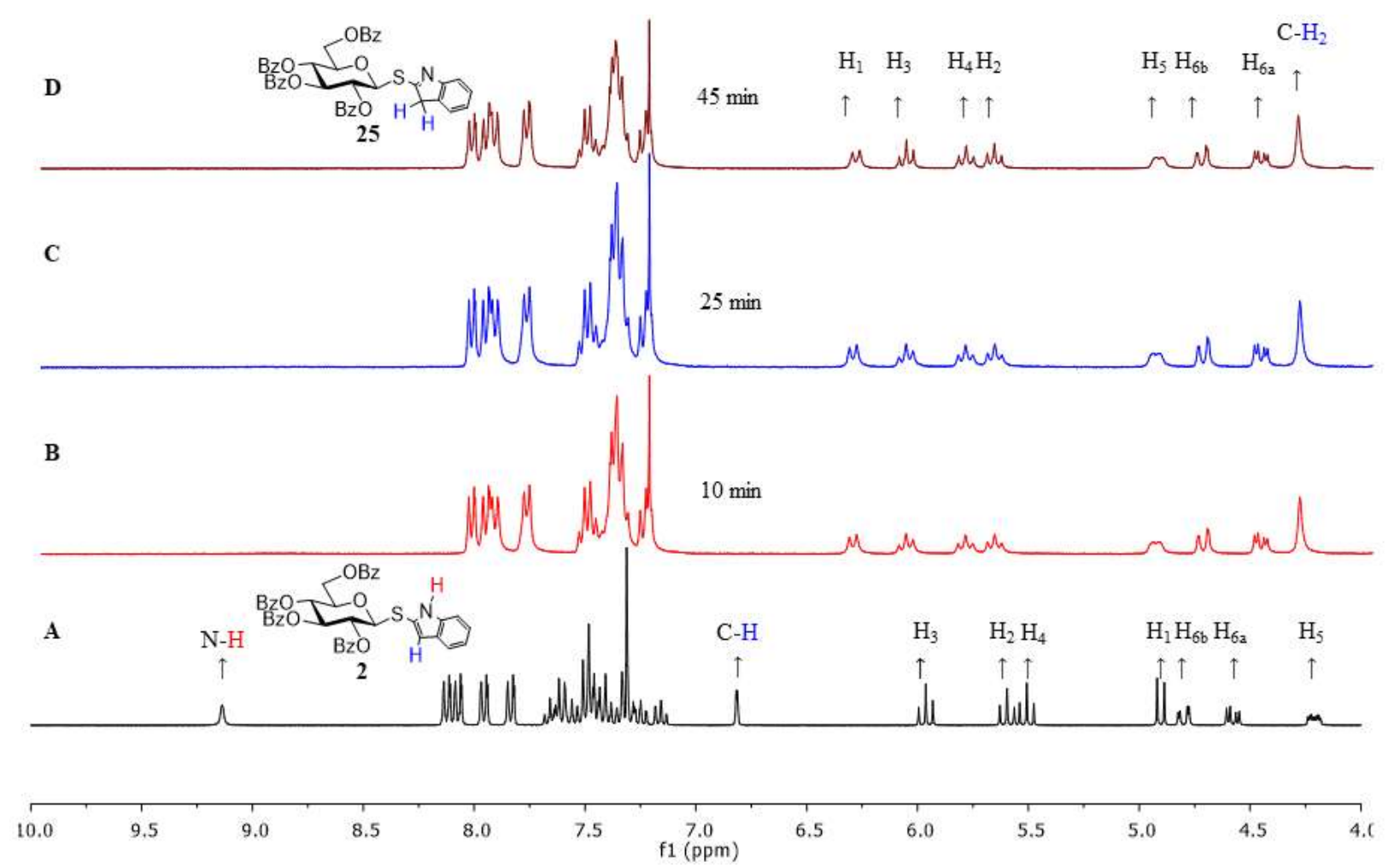

Figure S5: ${ }^{1} \mathrm{H}$ NMR spectra of A) glycosyl donor $\mathbf{2}$ in $\mathrm{CDCl}_{3}$; B) glycosyl donor $\mathbf{2}$ in $\mathrm{CDCl}_{3}$ in presence of 1 equiv TMSOTf at intervals of $10 \mathrm{~min}$; C) in $25 \mathrm{~min}$; D) in $45 \mathrm{~min}$.

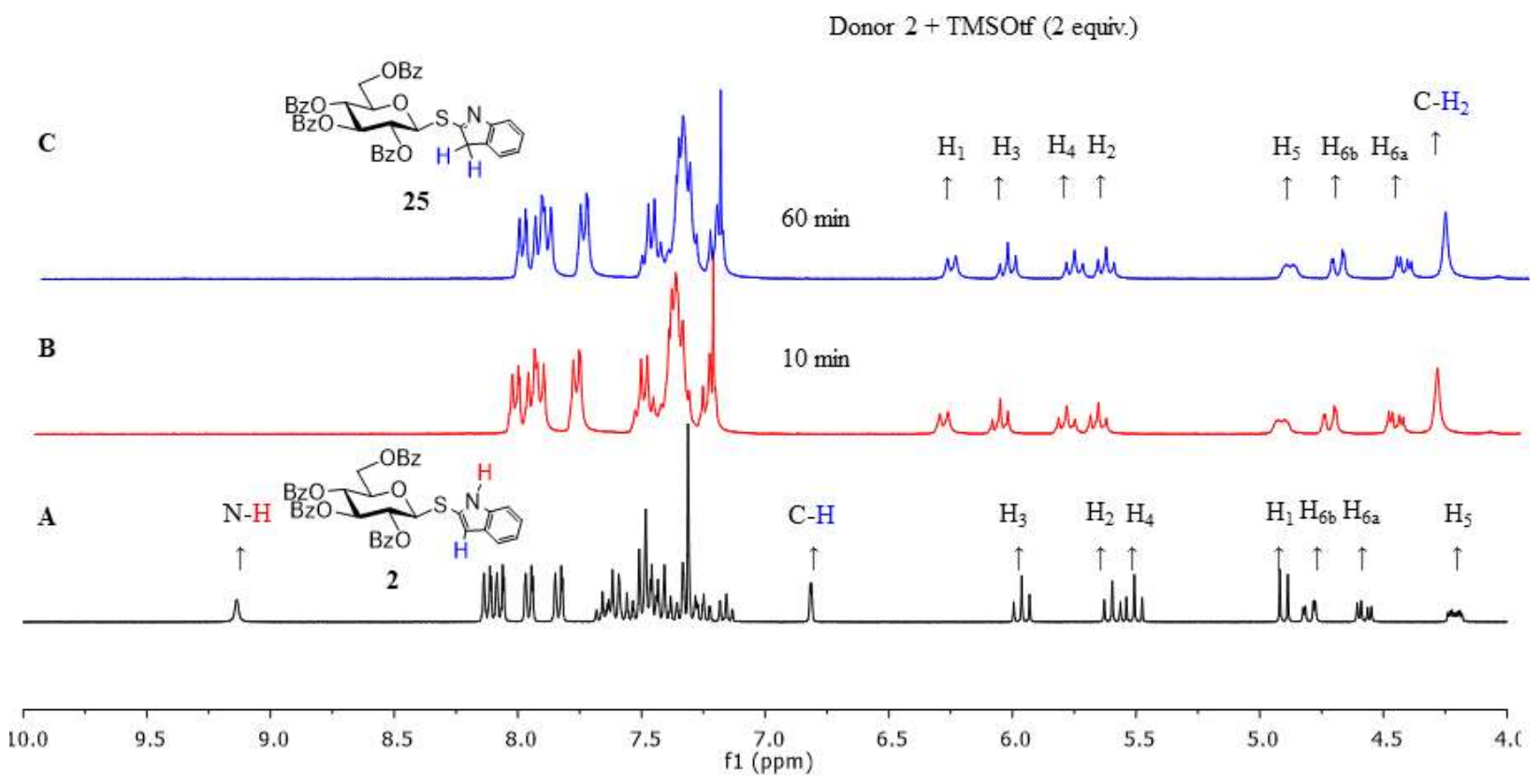

Figure S6: ${ }^{1} \mathrm{H}$ NMR spectra of A) glycosyl donor 2 in $\mathrm{CDCl}_{3}$; B) glycosyl donor $\mathbf{2}$ in $\mathrm{CDCl}_{3}$ in presence of 2 equiv TMSOTf at intervals of $10 \mathrm{~min}$; C) in $1 \mathrm{~h}$. 


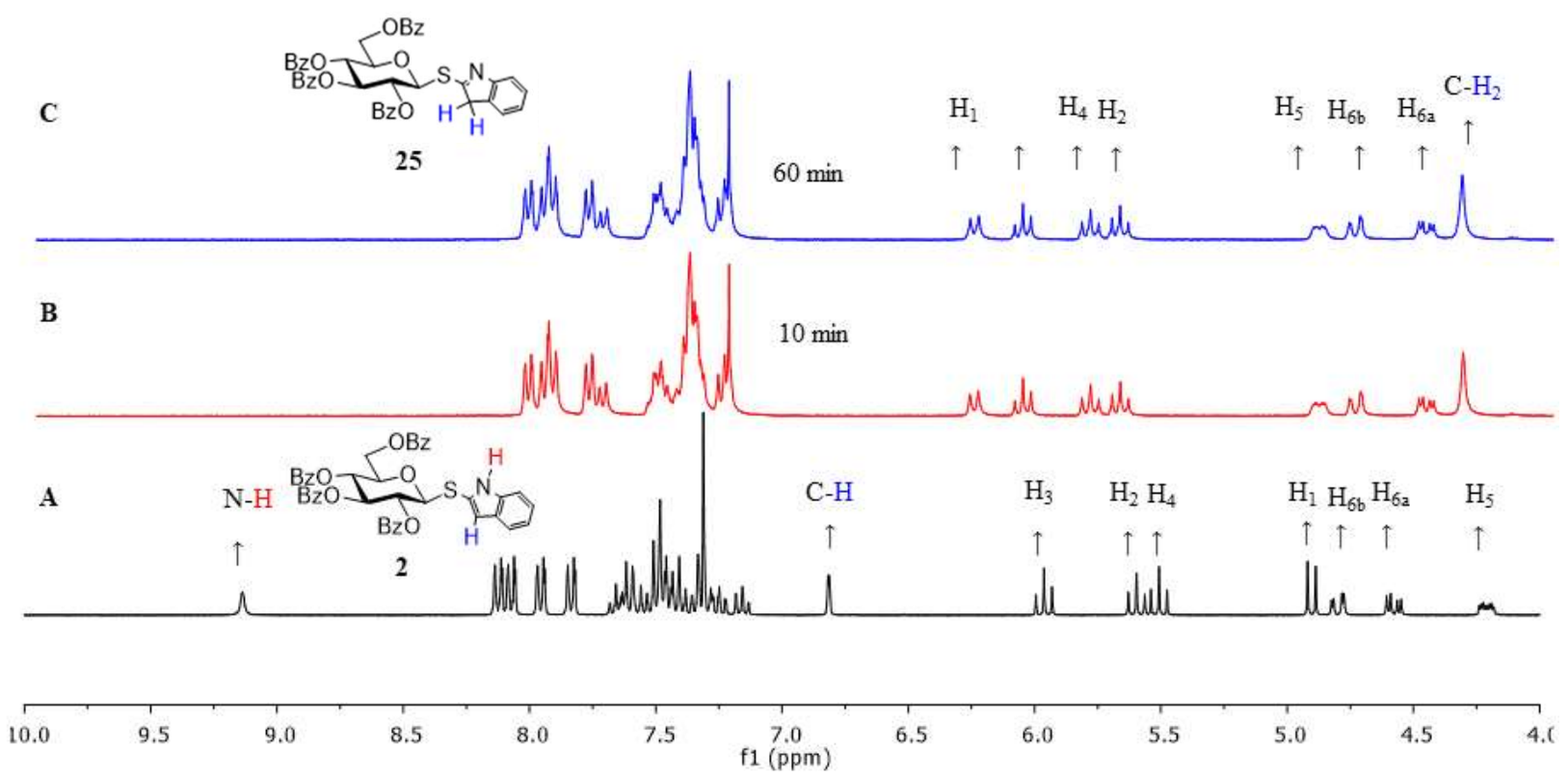

Figure S7: ${ }^{1} \mathrm{H}$ NMR spectra of A) glycosyl donor 2 in $\mathrm{CDCl}_{3}$; B) glycosyl donor 2 in $\mathrm{CDCl}_{3}$ in presence of 4 equiv of TMSOTf at intervals of $10 \mathrm{~min}$; C) in $1 \mathrm{~h}$.
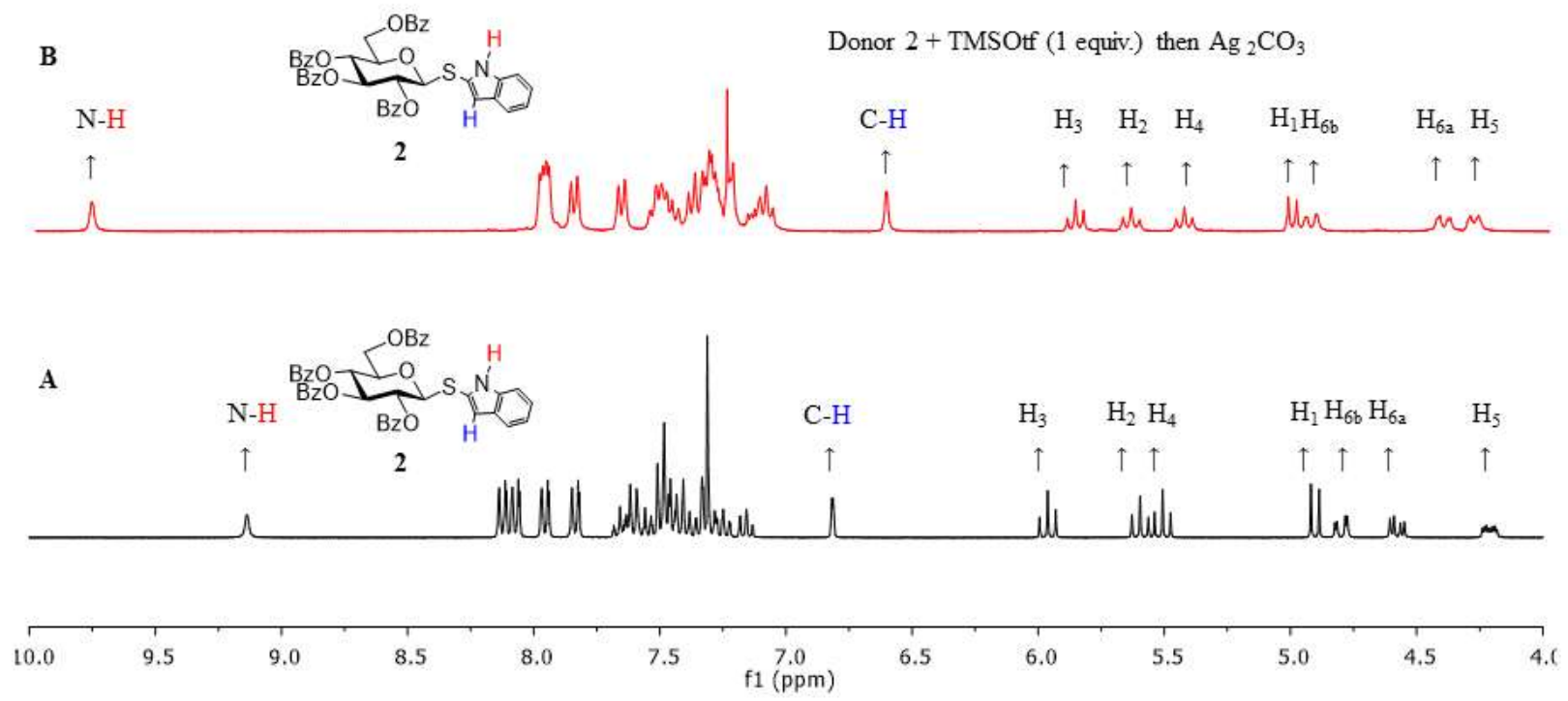

Figure S8: ${ }^{1} \mathrm{H}$ NMR spectra of A) glycosyl donor $\mathbf{2}$ in $\mathrm{CDCl}_{3}$; B) glycosyl donor $\mathbf{2}$ in $\mathrm{CDCl}_{3}$ in presence of 1 equiv. of TMSOTf and then $\mathrm{Ag}_{2} \mathrm{CO}_{3}$. 
Donor $2+$ TMSOtf (4 equiv.) then $\mathrm{Ag}_{2} \mathrm{CO}_{3}$

B

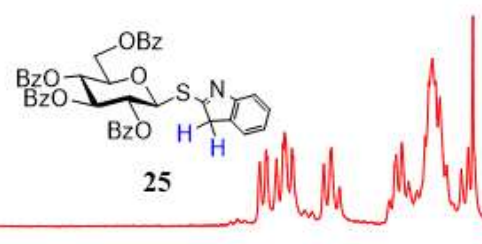

$\begin{array}{llll}\mathrm{H}_{1} & \mathrm{H}_{3} & \mathrm{H}_{4} & \mathrm{H}_{2}\end{array}$

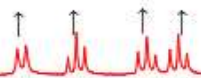

$\begin{array}{llll}\mathrm{H}_{5} & \mathrm{H}_{6 \mathrm{~b}} & \mathrm{H}_{6 \mathrm{a}} & \uparrow\end{array}$

C- $\mathrm{H}_{2}$

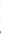
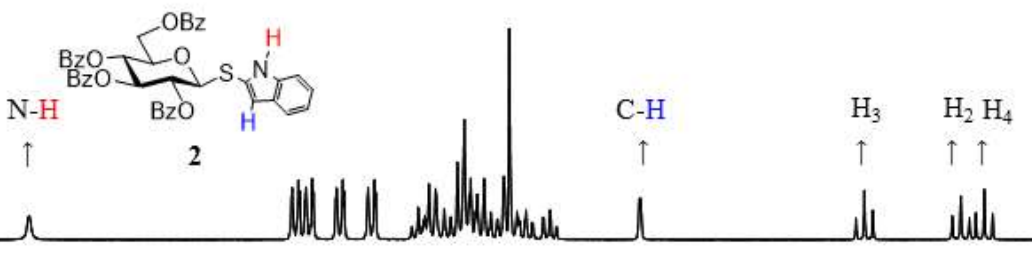

$\begin{array}{llll}\mathrm{H}_{1} \mathrm{H}_{6 \mathrm{~b}} & \mathrm{H}_{6 \mathrm{a}} & \mathrm{H}_{5}\end{array}$

$\uparrow \uparrow \uparrow \uparrow$

$\Lambda$

M. Whow Whath

alda
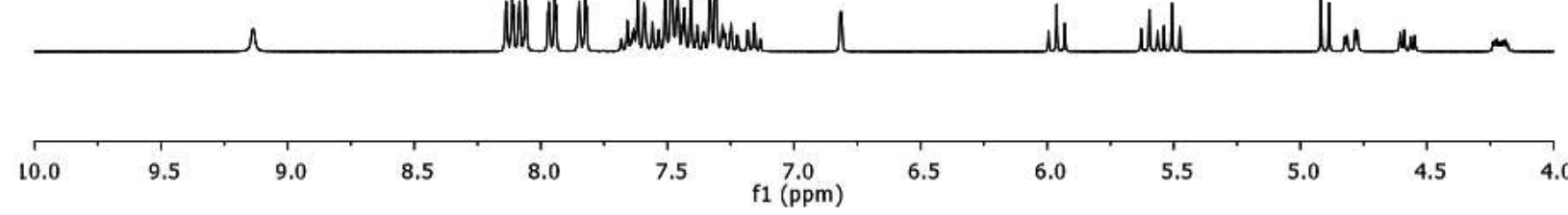

Figure S9: ${ }^{1} \mathrm{H}$ NMR spectra of A) glycosyl donor 2 in $\mathrm{CDCl}_{3}$; B) glycosyl donor $\mathbf{2}$ in $\mathrm{CDCl}_{3}$ in presence of 4 equiv TMSOTf and then $\mathrm{Ag}_{2} \mathrm{CO}_{3}$.

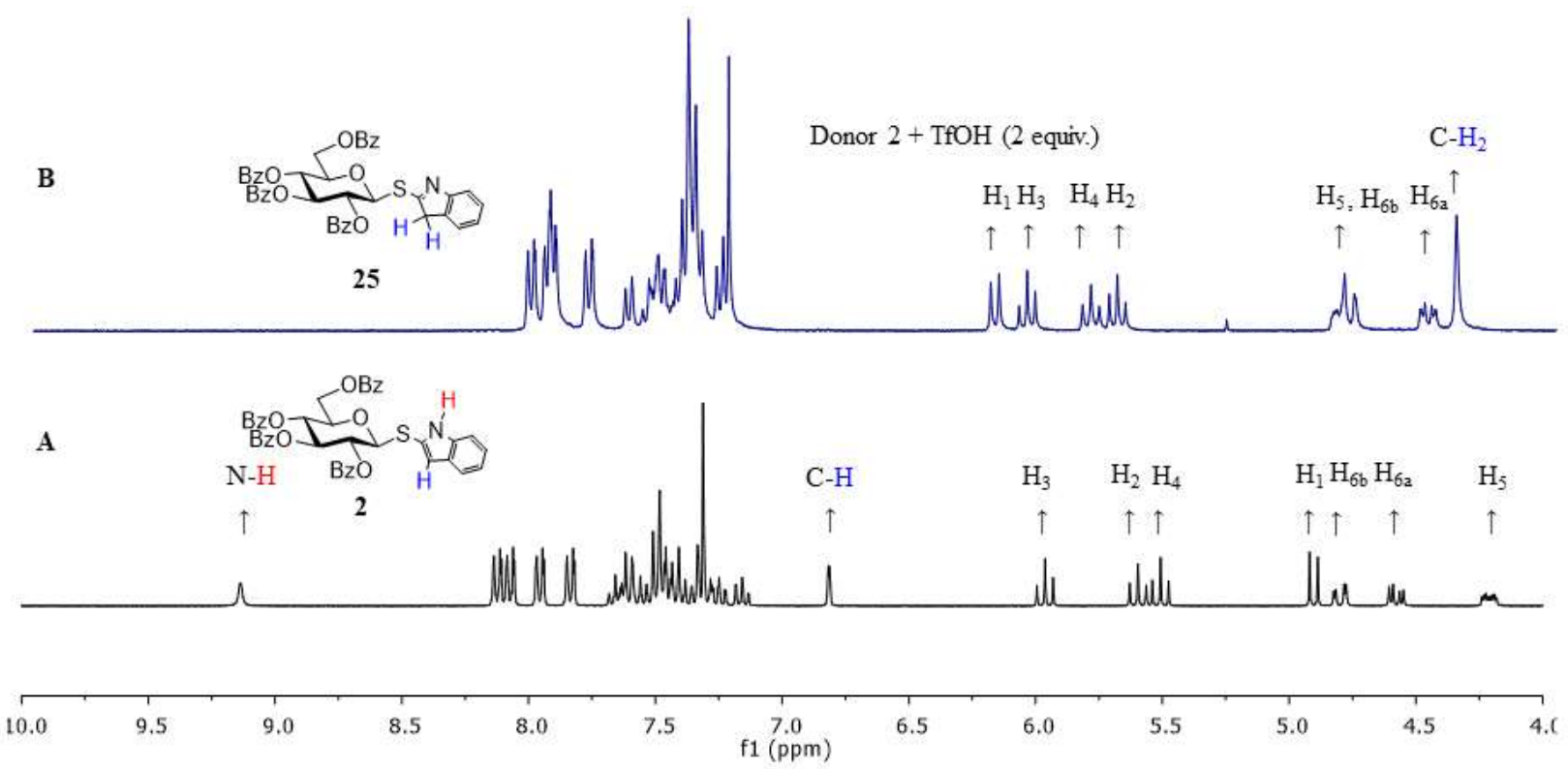

Figure S10: ${ }^{1} \mathrm{H}$ NMR spectra of A) glycosyl donor 2 in $\mathrm{CDCl}_{3}$; B) glycosyl donor $\mathbf{2}$ in $\mathrm{CDCl}_{3}$ in presence of 2 equiv $\mathrm{TfOH}$. 
NMR Spectra of new compounds

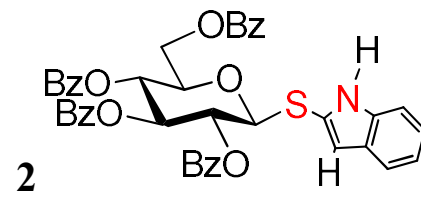

${ }^{1} \mathrm{H} \mathrm{NMR}\left(\mathrm{CDCl}_{3}, 300 \mathrm{MHz}\right)$

ஜ

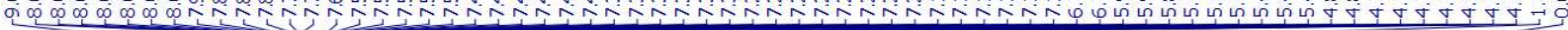

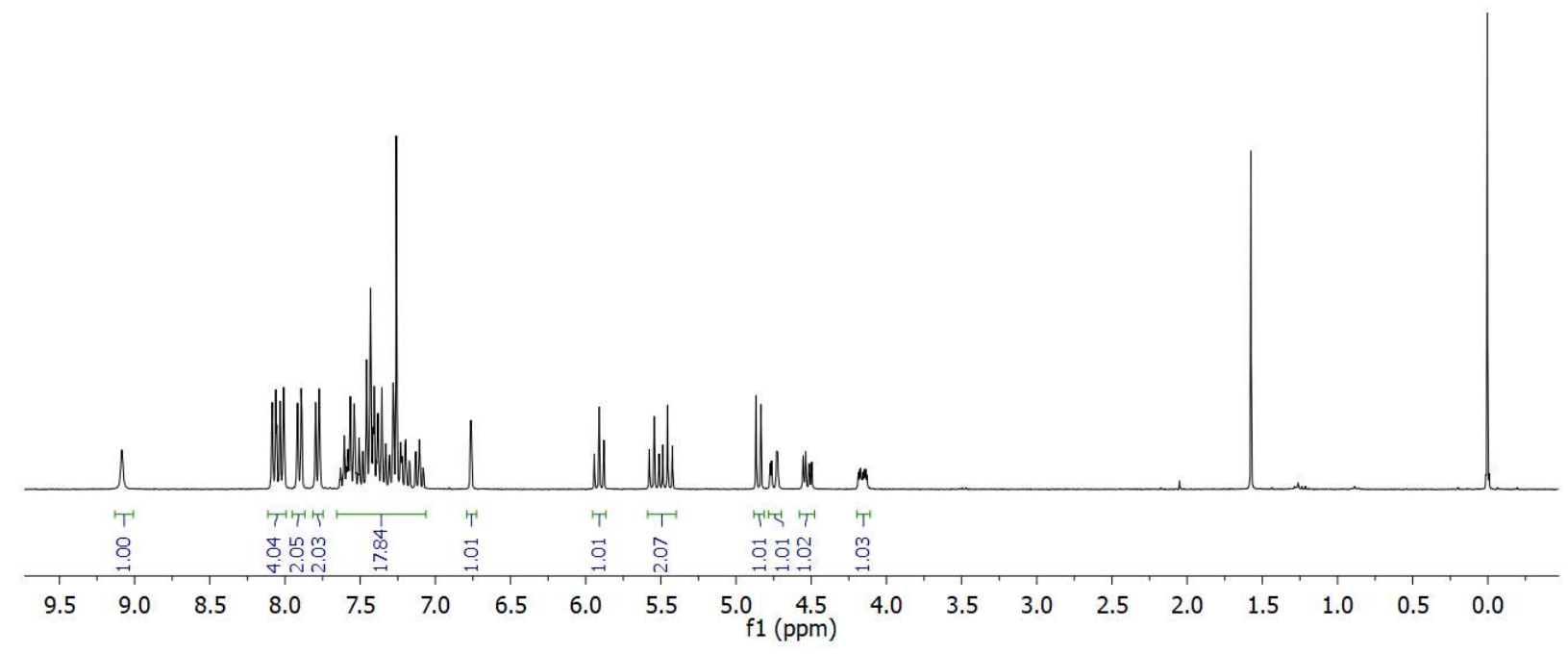




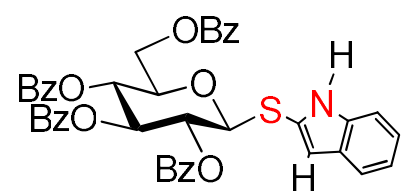

2

$\mathrm{COSY}\left(\mathrm{CDCl}_{3}, 300 \mathrm{MHz}\right)$

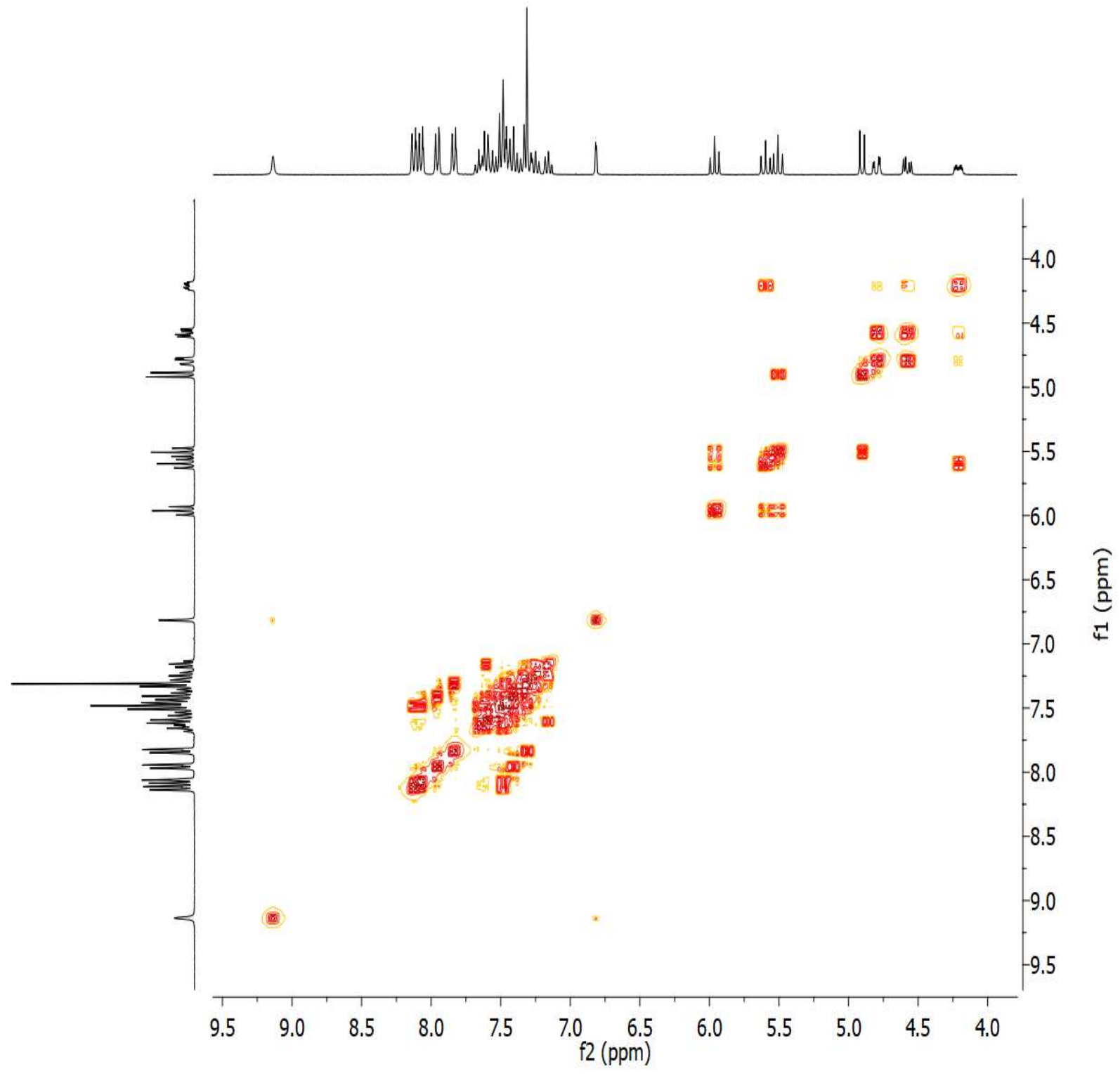




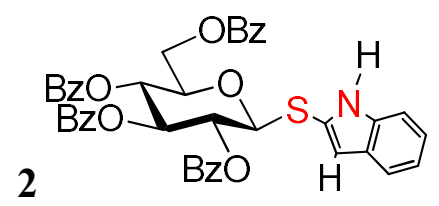

${ }^{13} \mathrm{C} \mathrm{NMR}\left(\mathrm{CDCl}_{3}, 75 \mathrm{MHz}\right)$

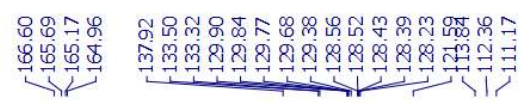

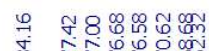

눈

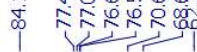

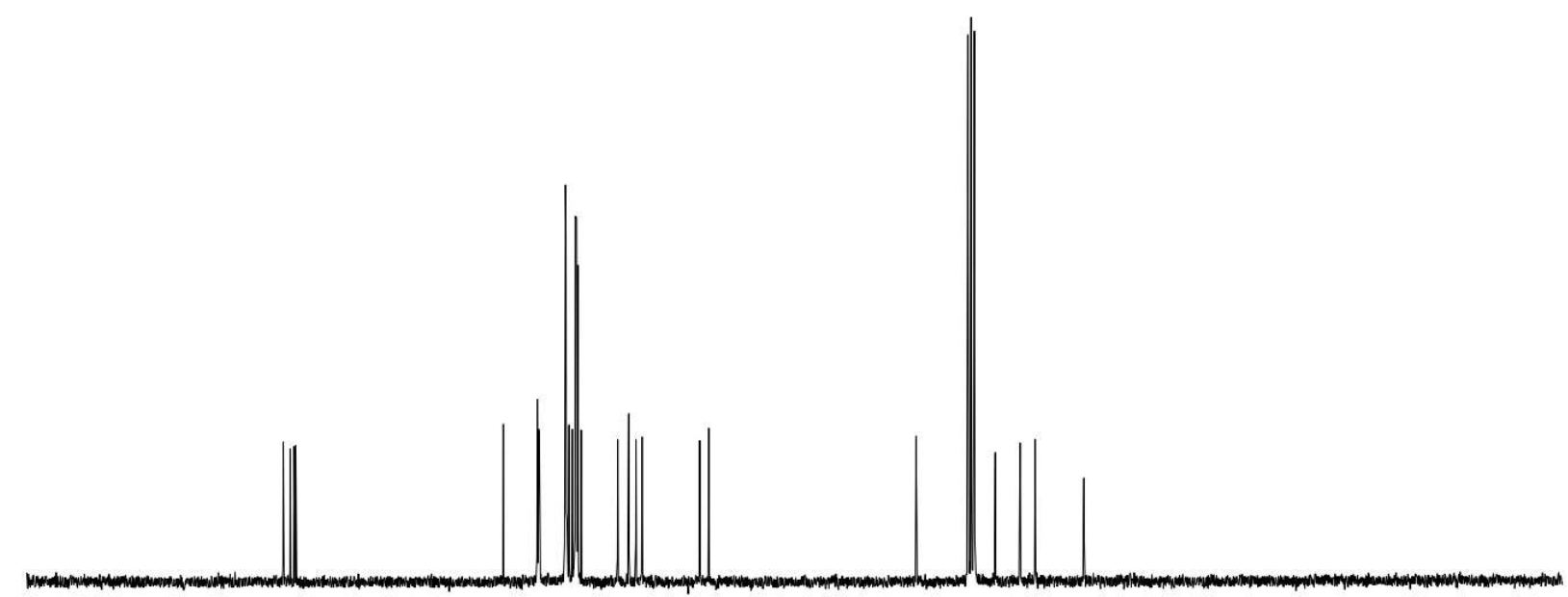

200

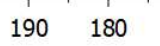

$170 \quad 160$

150

140130

120

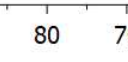




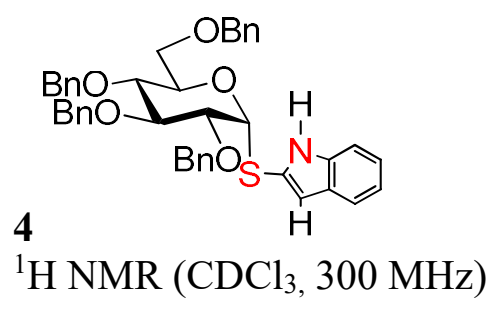

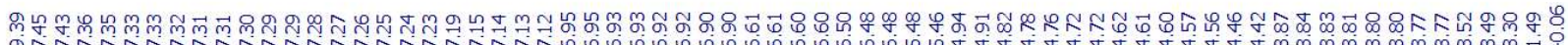

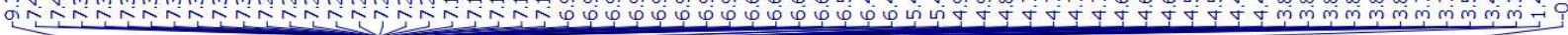

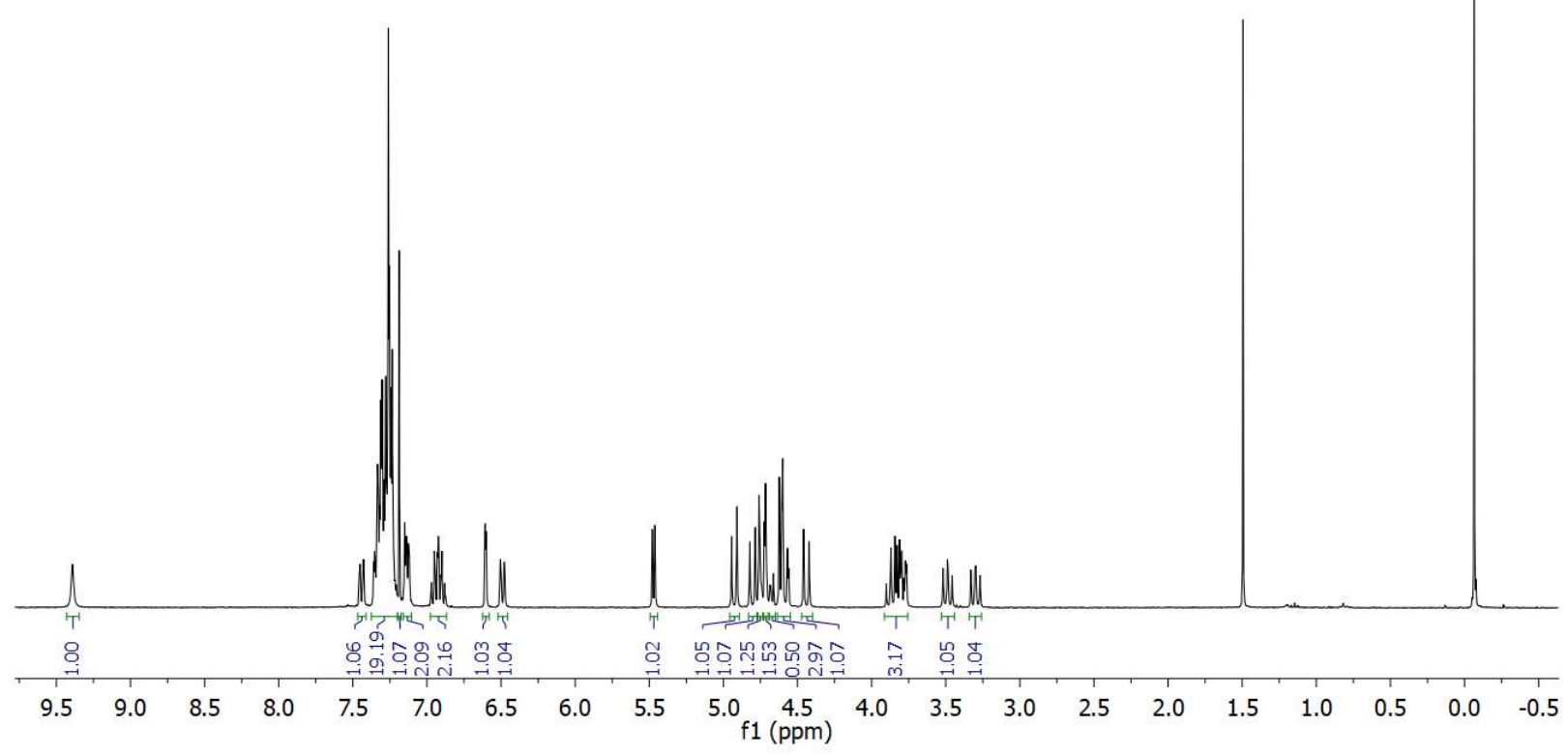
333 

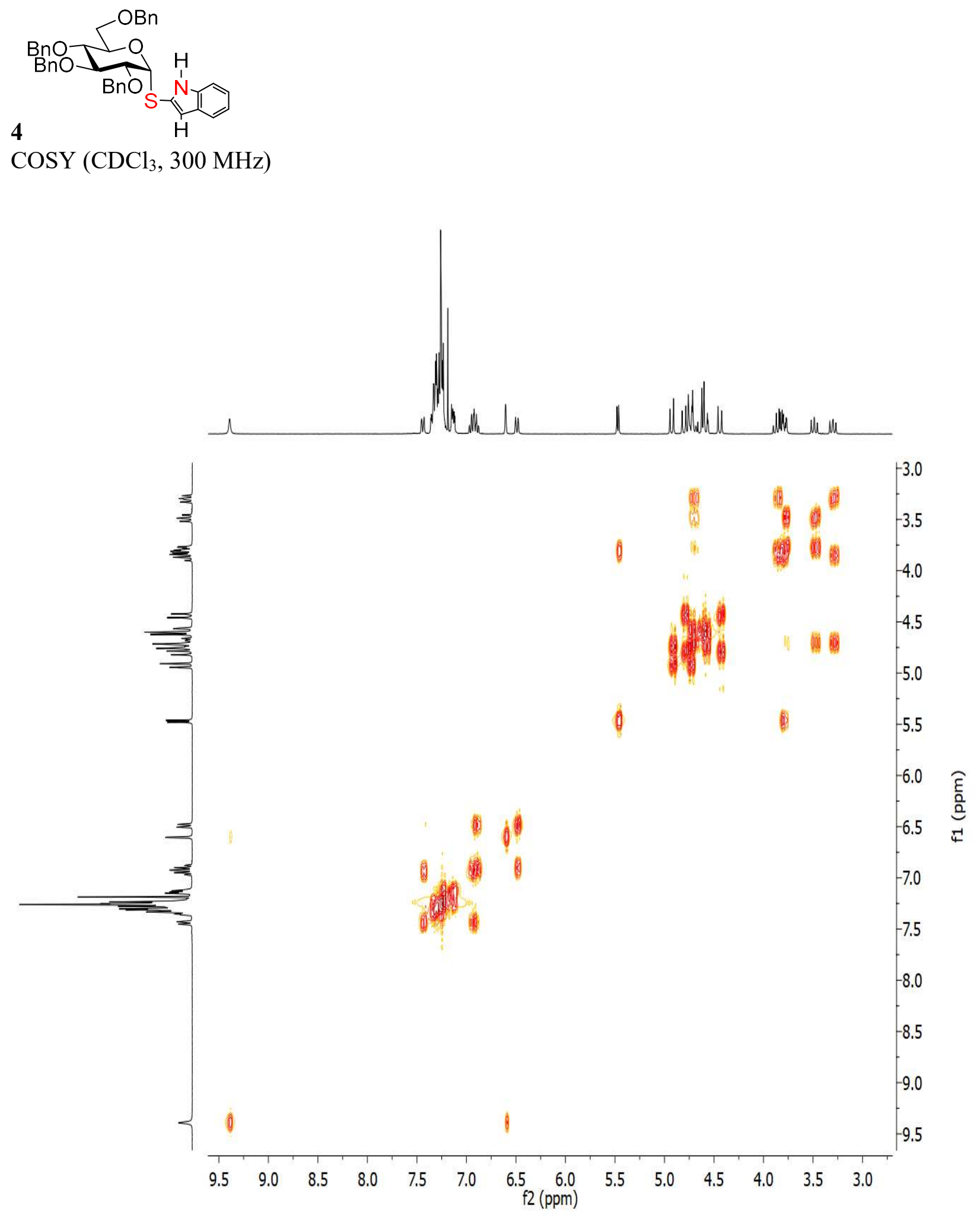


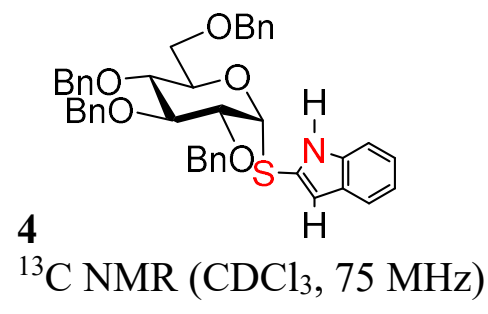

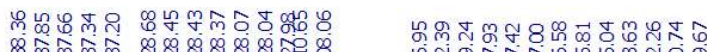

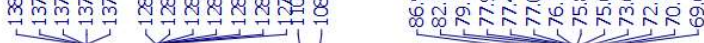

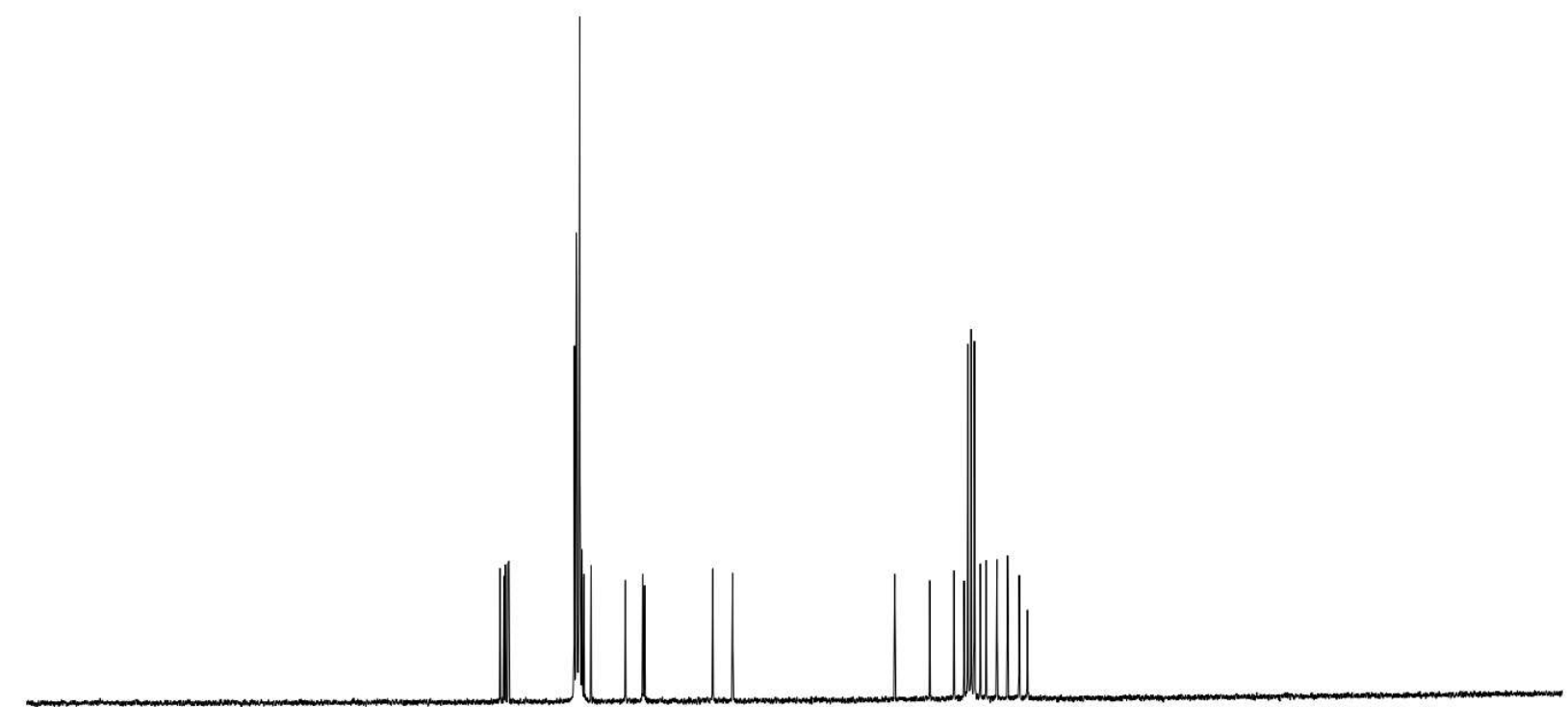

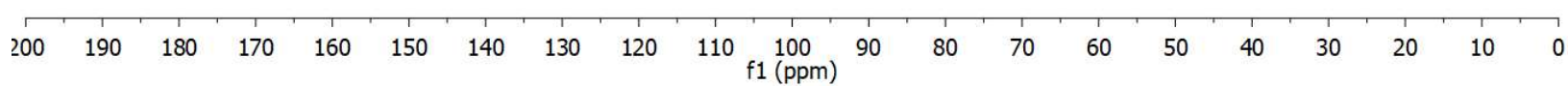




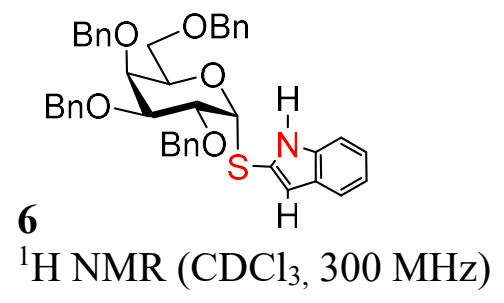

क ๆ

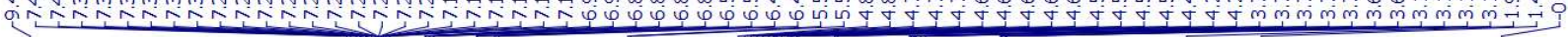

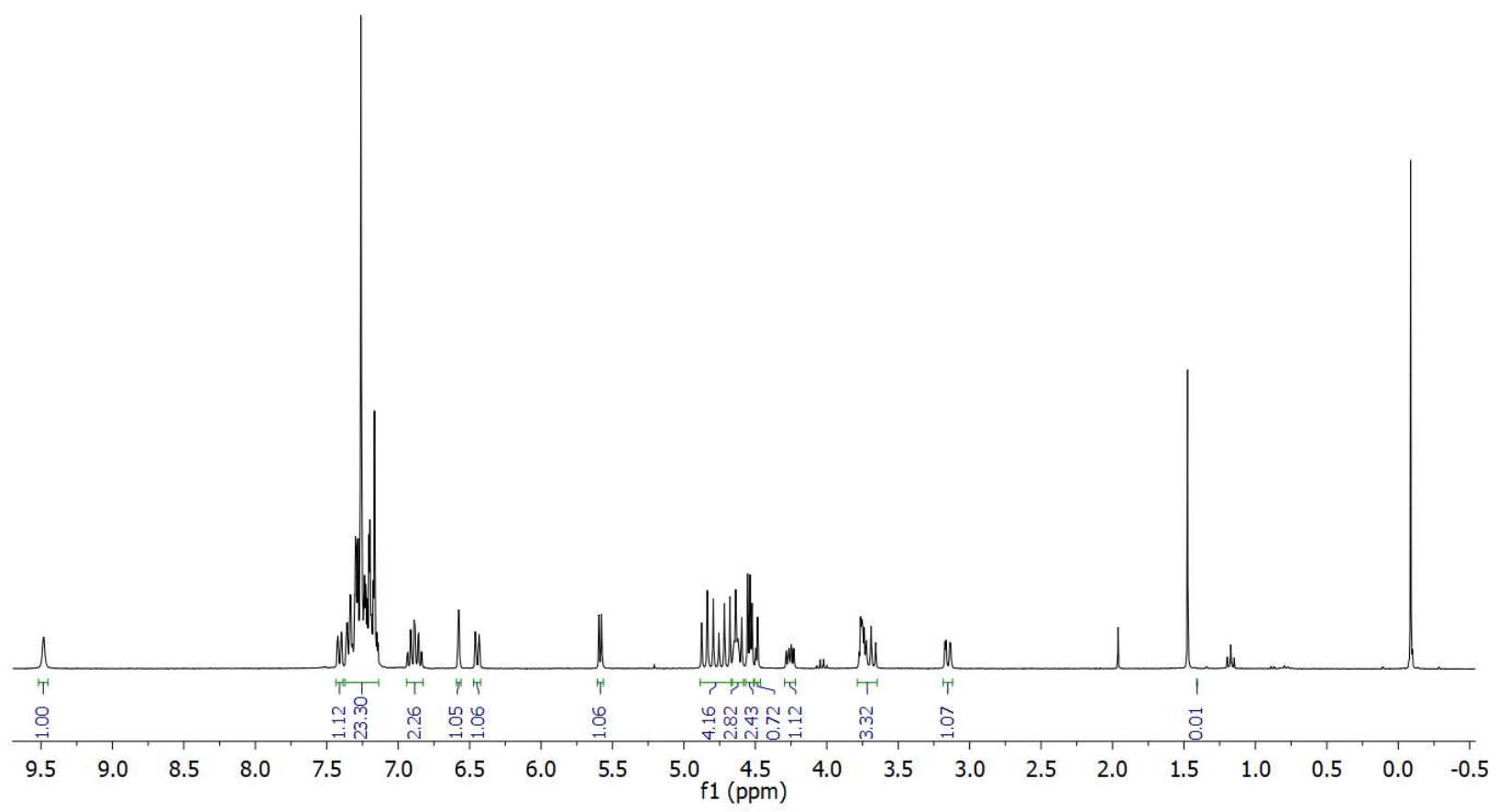



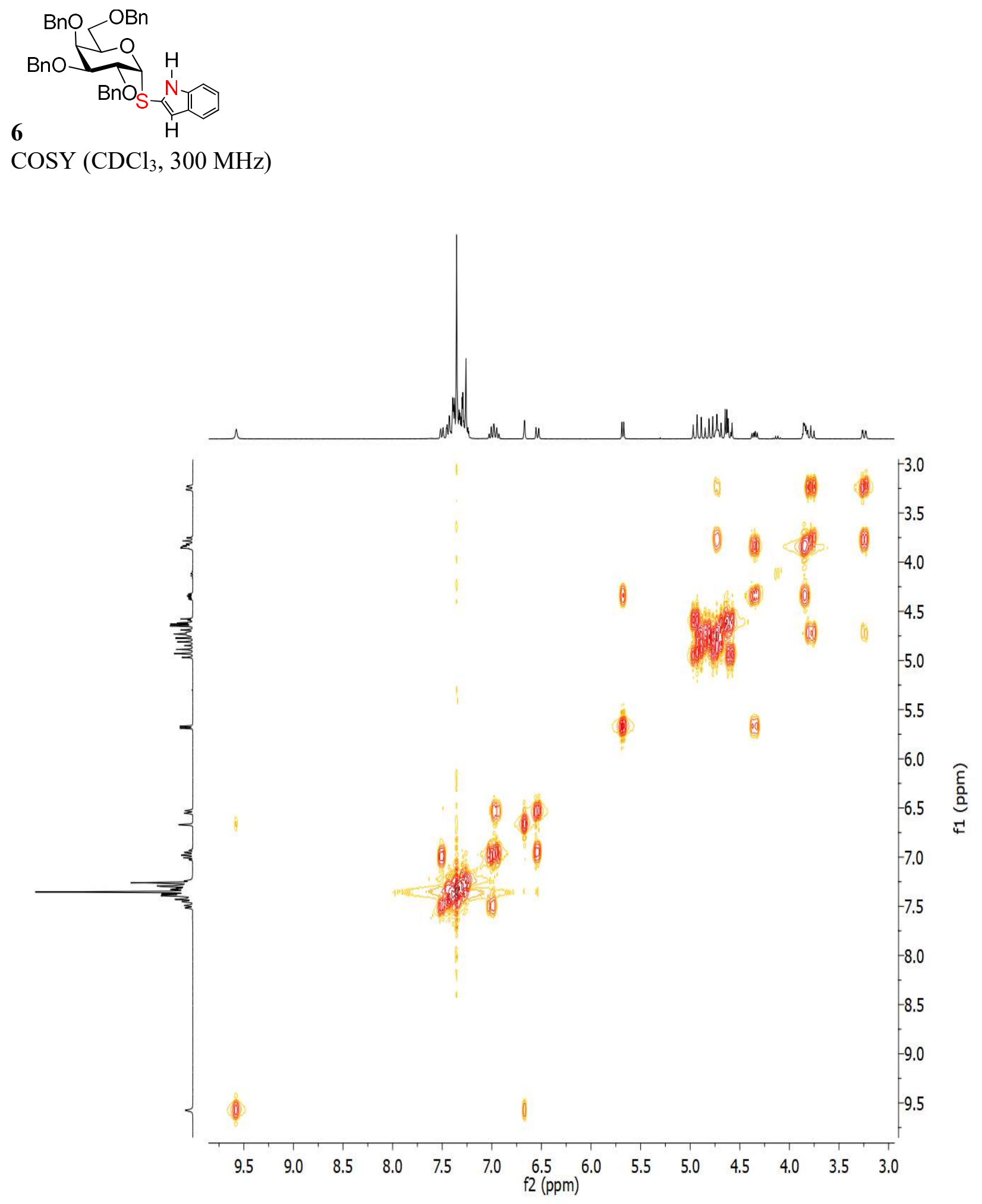
${ }^{13} \mathrm{C} \mathrm{NMR}\left(\mathrm{CDCl}_{3}, 75 \mathrm{MHz}\right)$

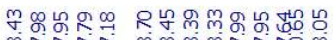

舟兹向

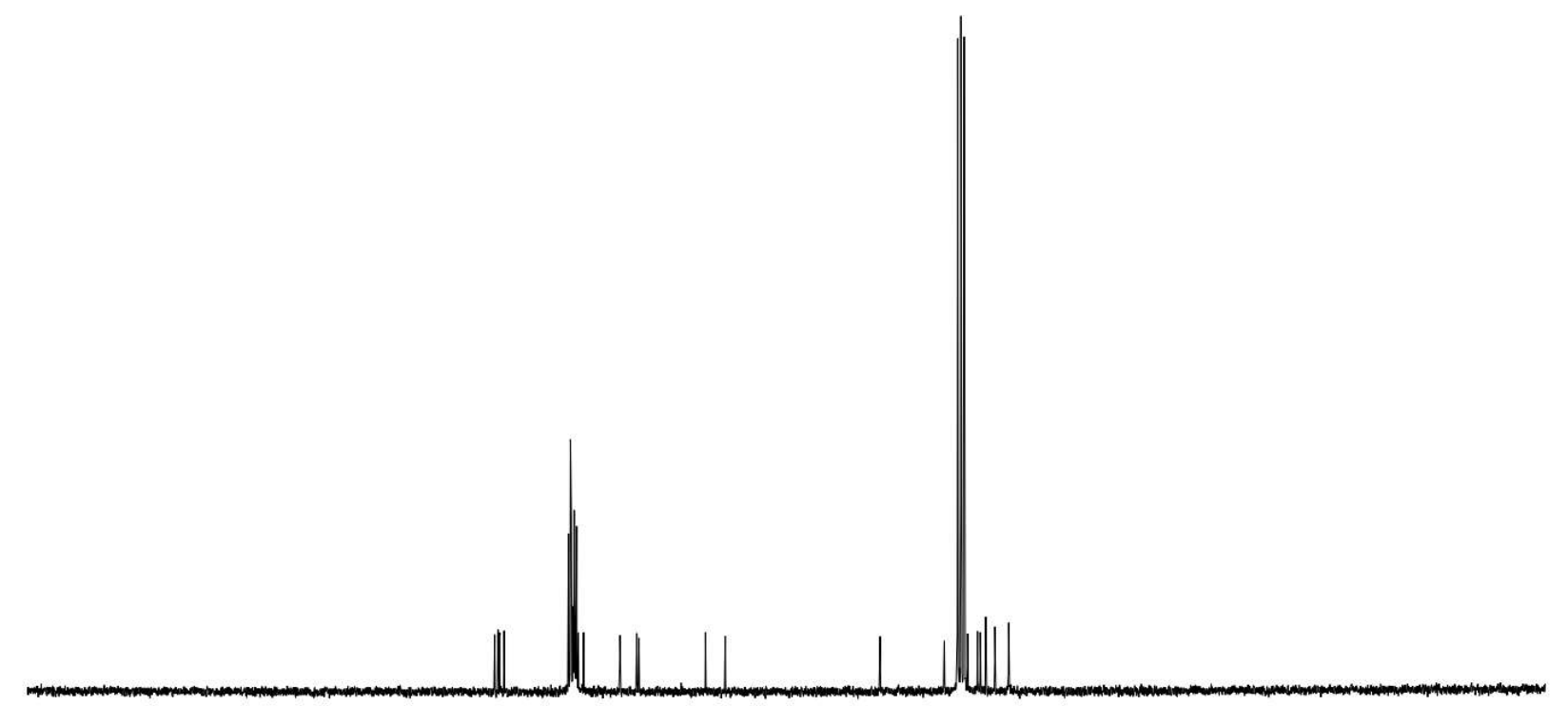


$\underline{\text { NMR spectra of recovered products from competition experiments }}$

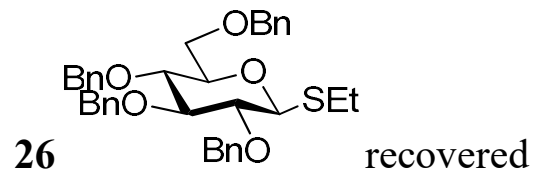

${ }^{1} \mathrm{H} \mathrm{NMR}\left(\mathrm{CDCl}_{3}, 300 \mathrm{MHz}\right)$

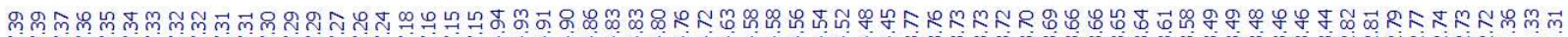

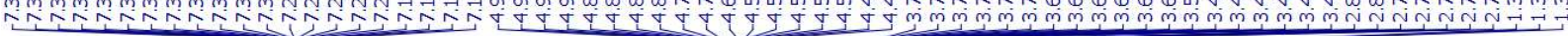

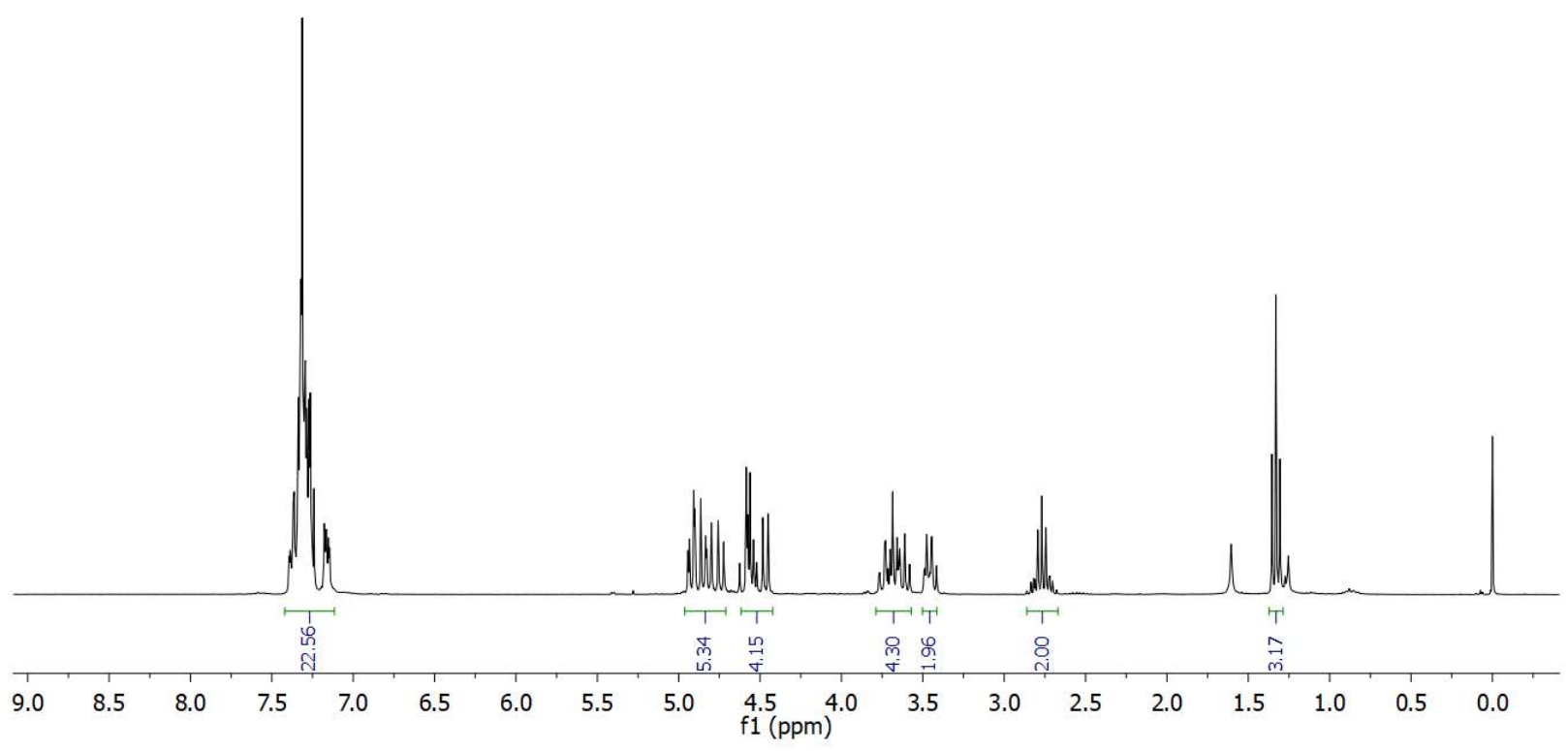




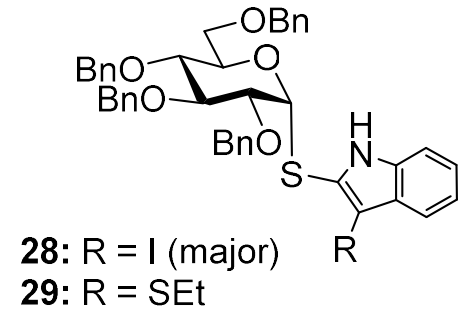

${ }^{1} \mathrm{H} \mathrm{NMR}\left(\mathrm{CDCl}_{3}, 300 \mathrm{MHz}\right)$

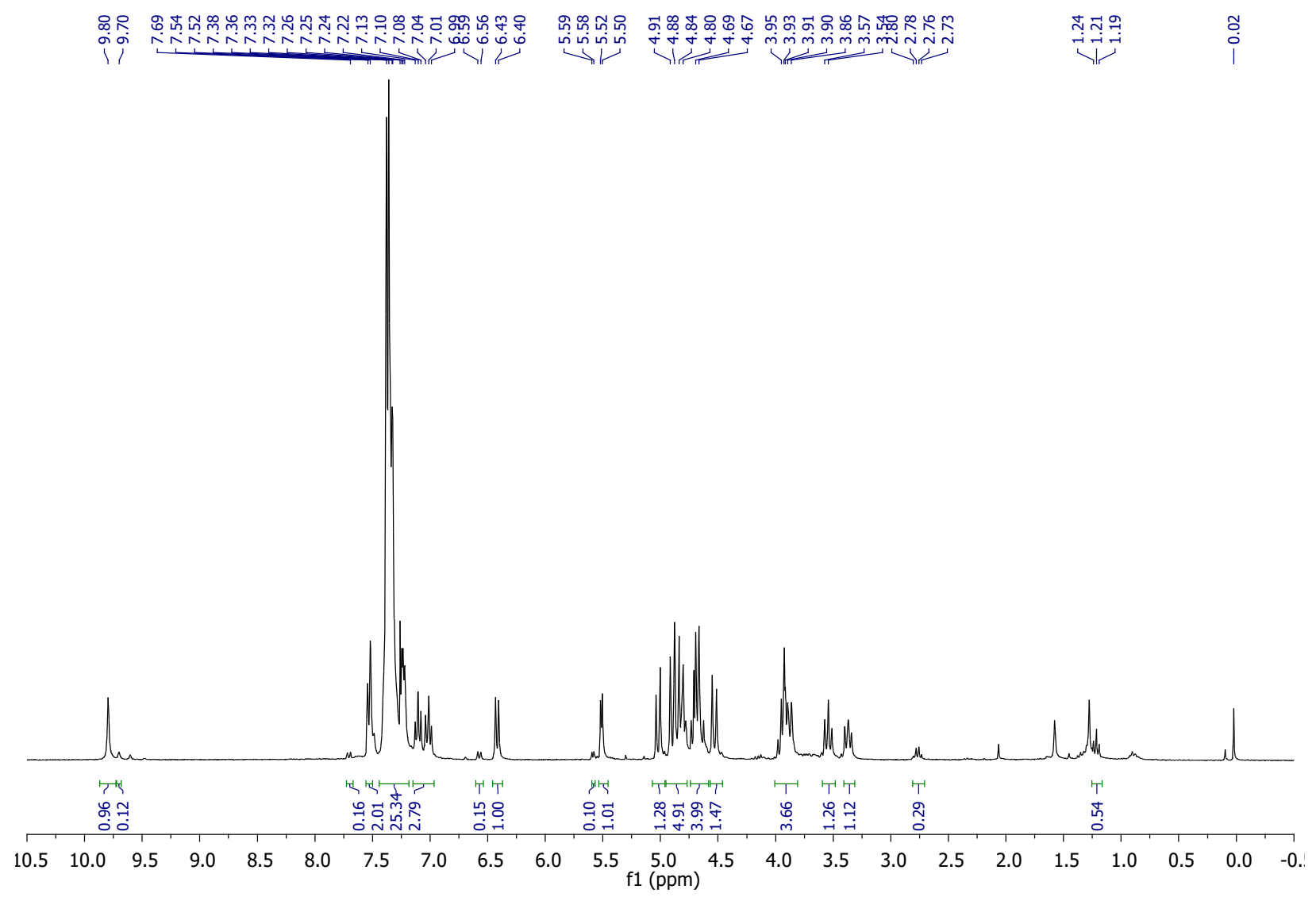


NMR Spectra of known disaccharides

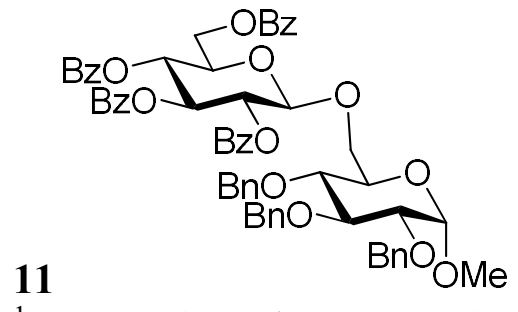

${ }^{1} \mathrm{H} \mathrm{NMR}\left(\mathrm{CDCl}_{3}, 300 \mathrm{MHz}\right)$

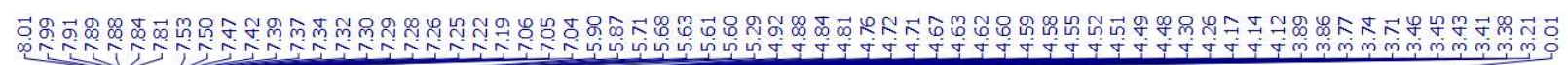

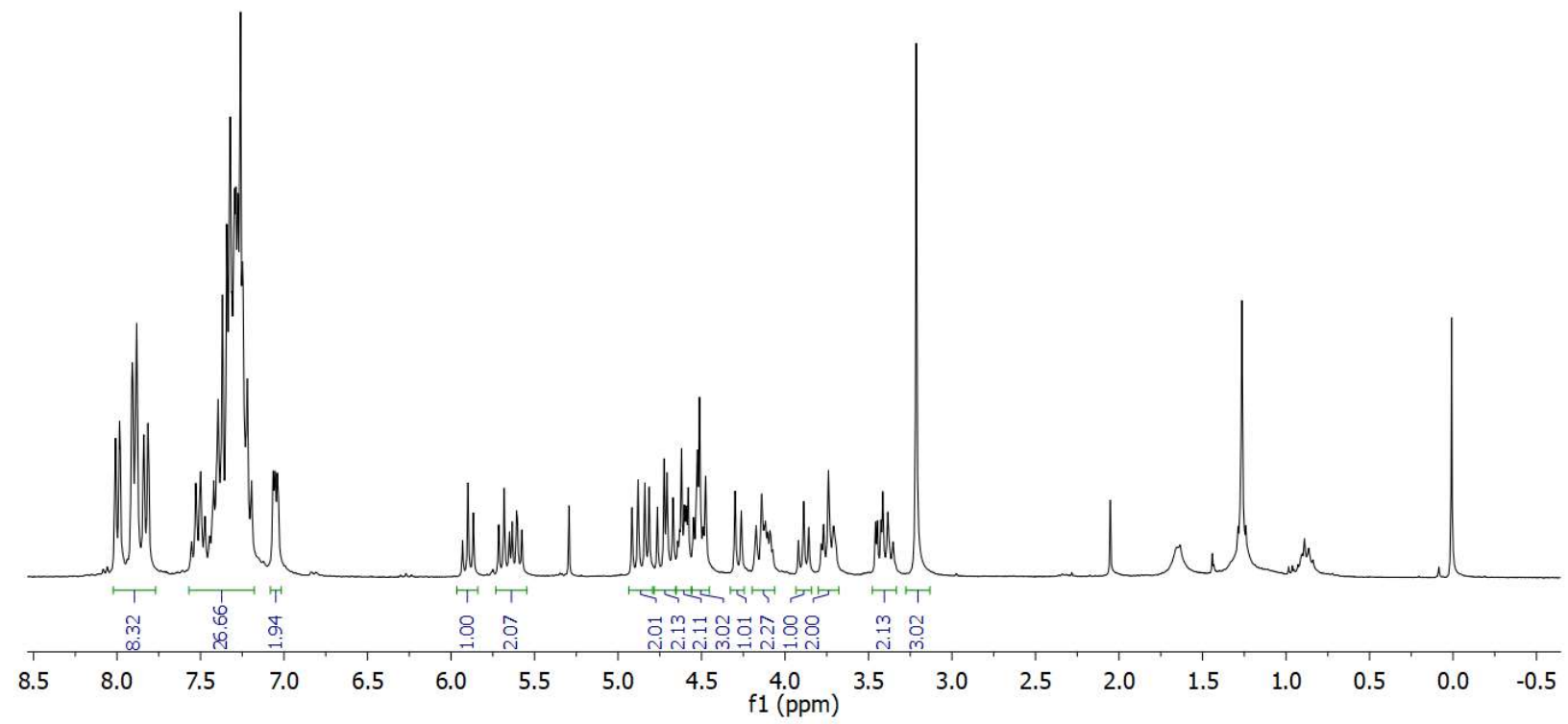




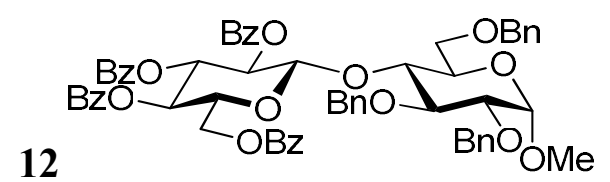

${ }^{1} \mathrm{H} \mathrm{NMR}\left(\mathrm{CDCl}_{3}, 300 \mathrm{MHz}\right)$

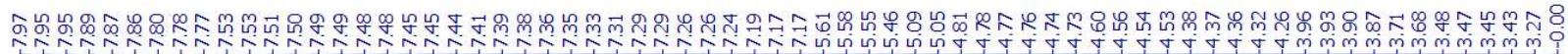

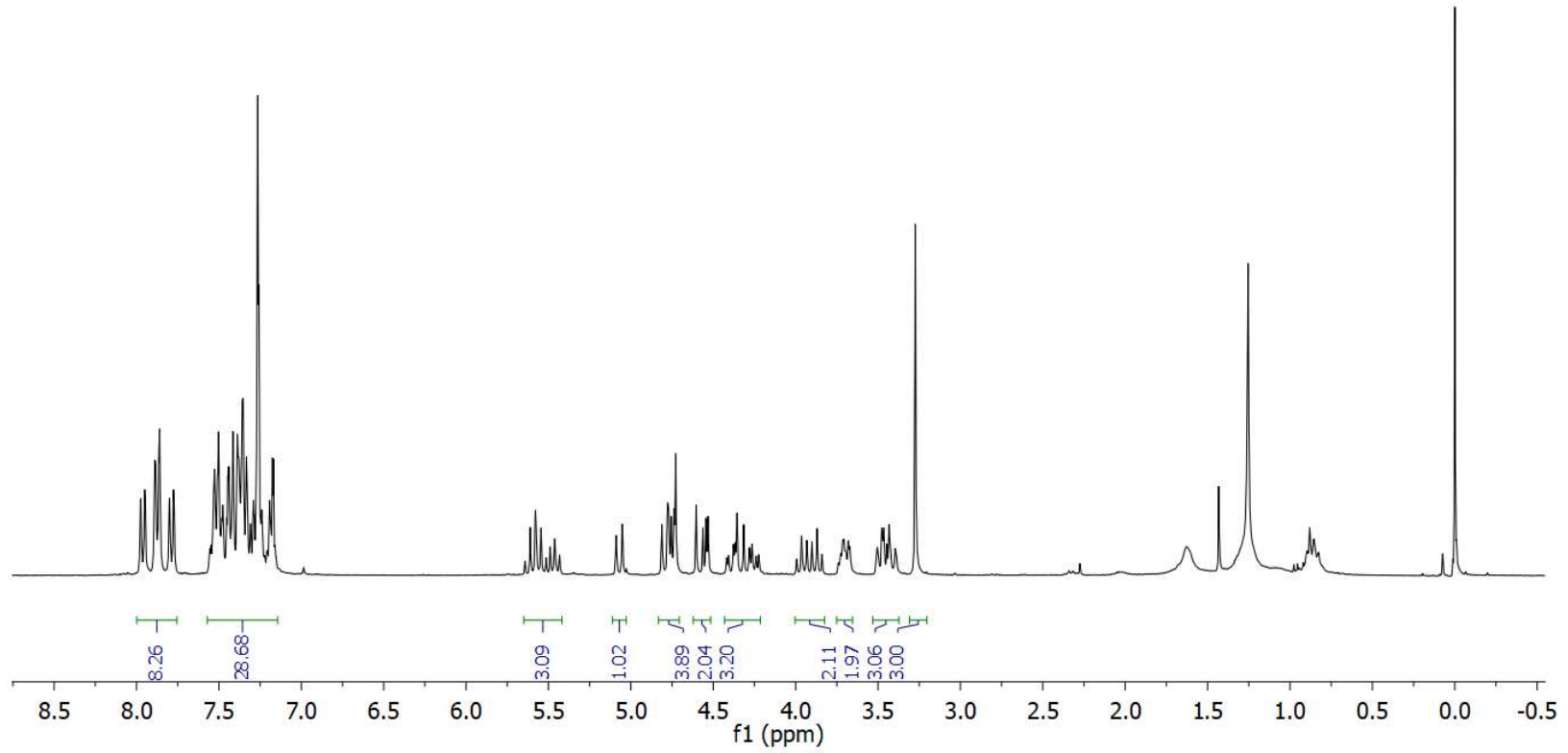




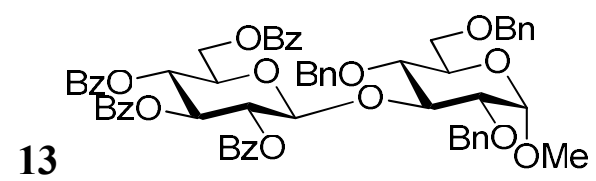

${ }^{1} \mathrm{H} \mathrm{NMR}\left(\mathrm{CDCl}_{3}, 300 \mathrm{MHz}\right)$
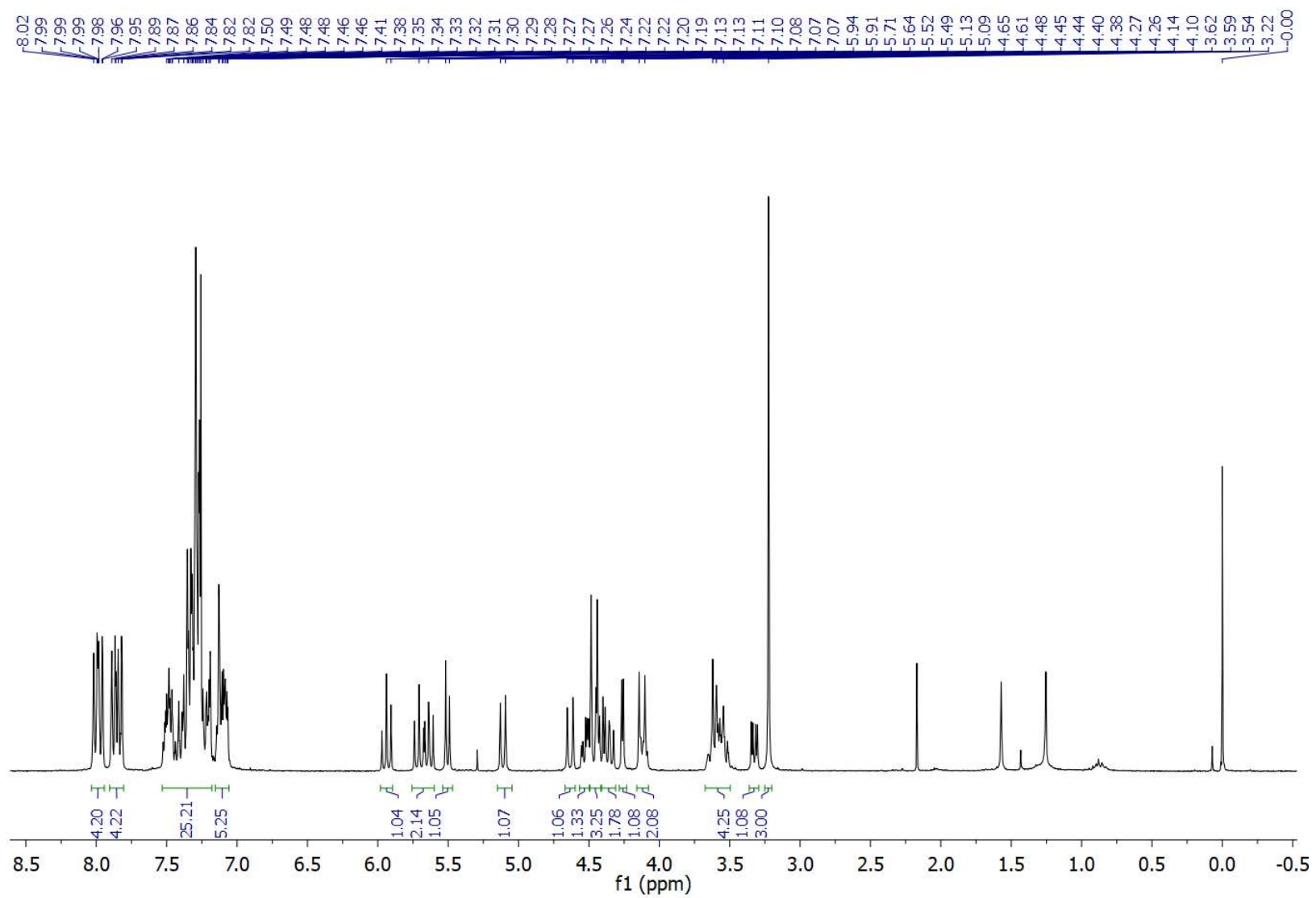


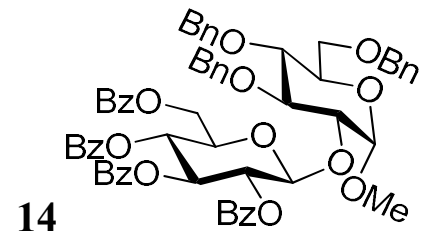

${ }^{1} \mathrm{H} \mathrm{NMR}\left(\mathrm{CDCl}_{3}, 300 \mathrm{MHz}\right)$

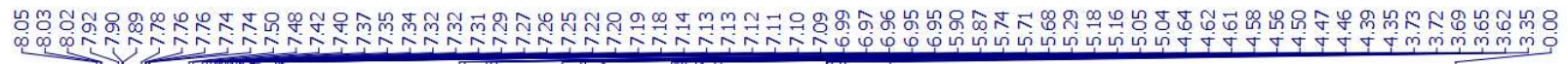

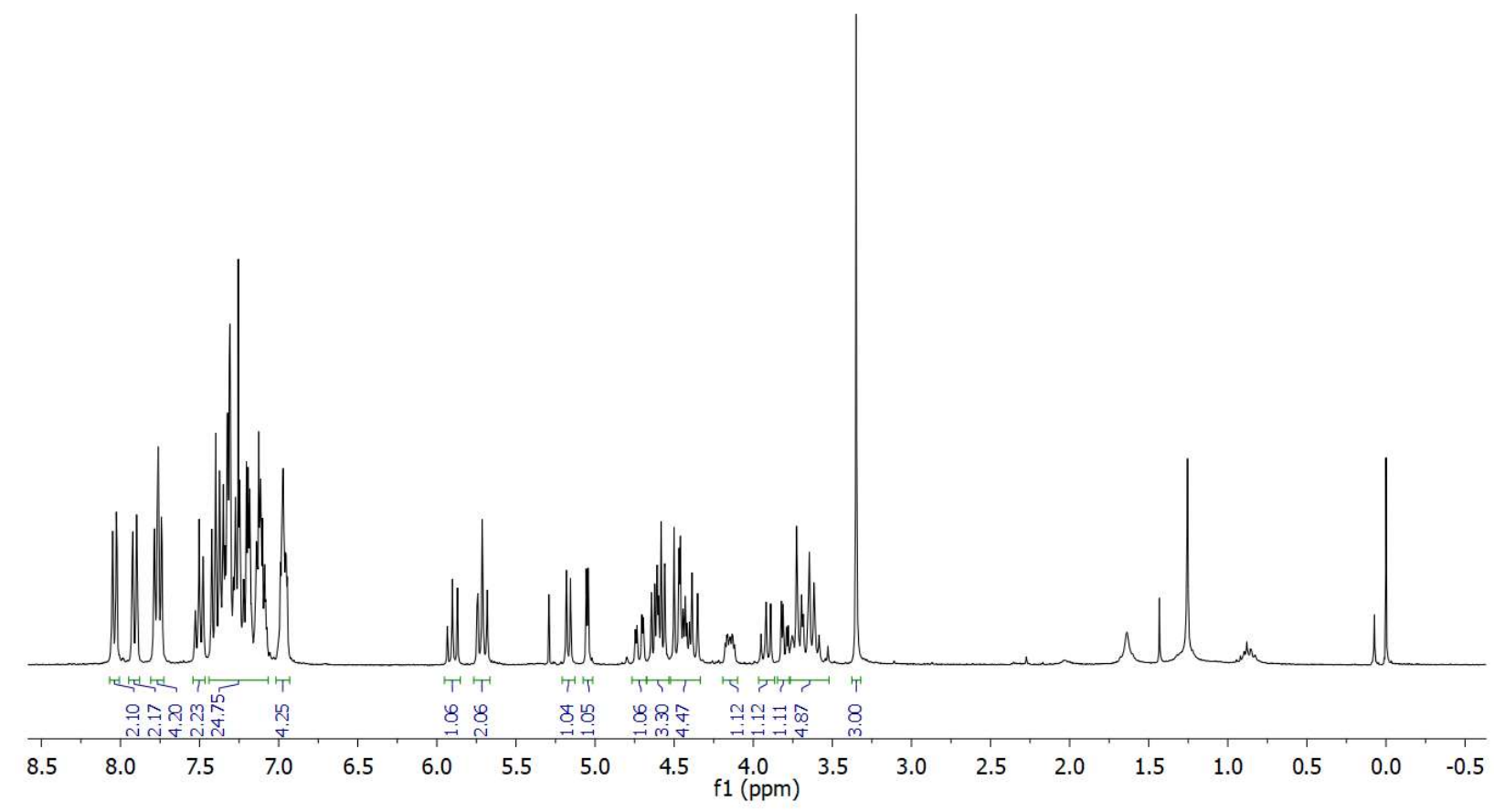




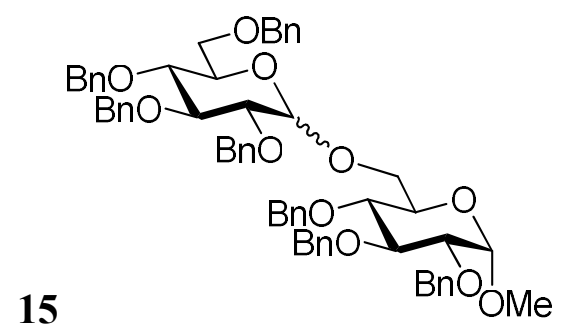

${ }^{1} \mathrm{H} \mathrm{NMR}\left(\mathrm{CDCl}_{3}, 300 \mathrm{MHz}\right)$

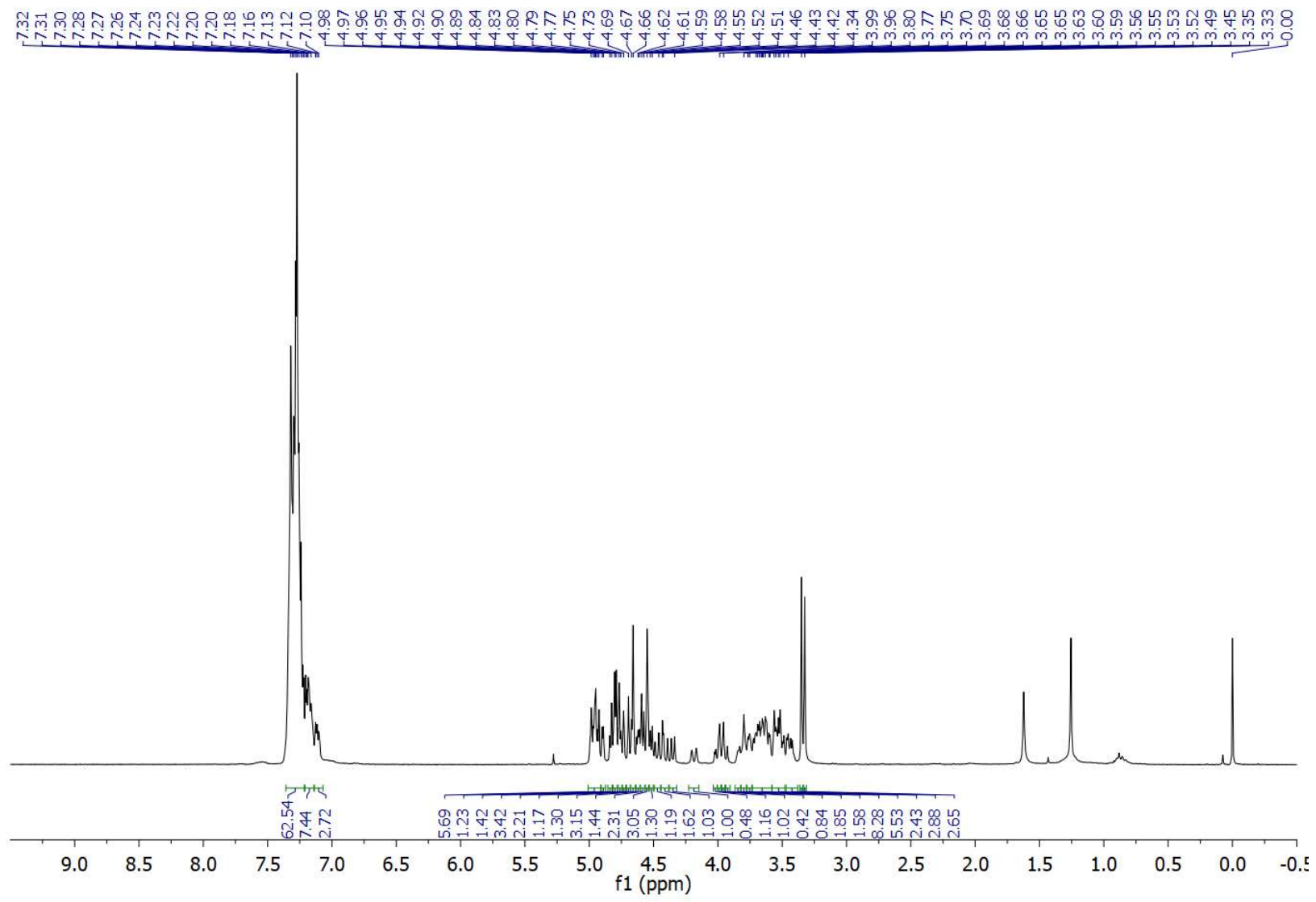




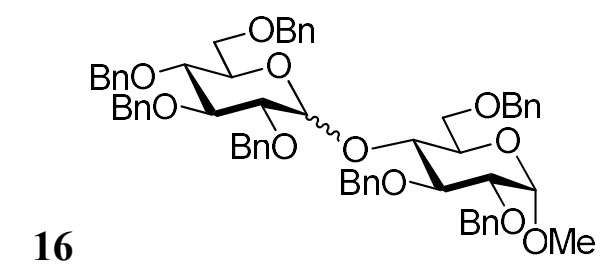

${ }^{1} \mathrm{H} \mathrm{NMR}\left(\mathrm{CDCl}_{3}, 300 \mathrm{MHz}\right)$

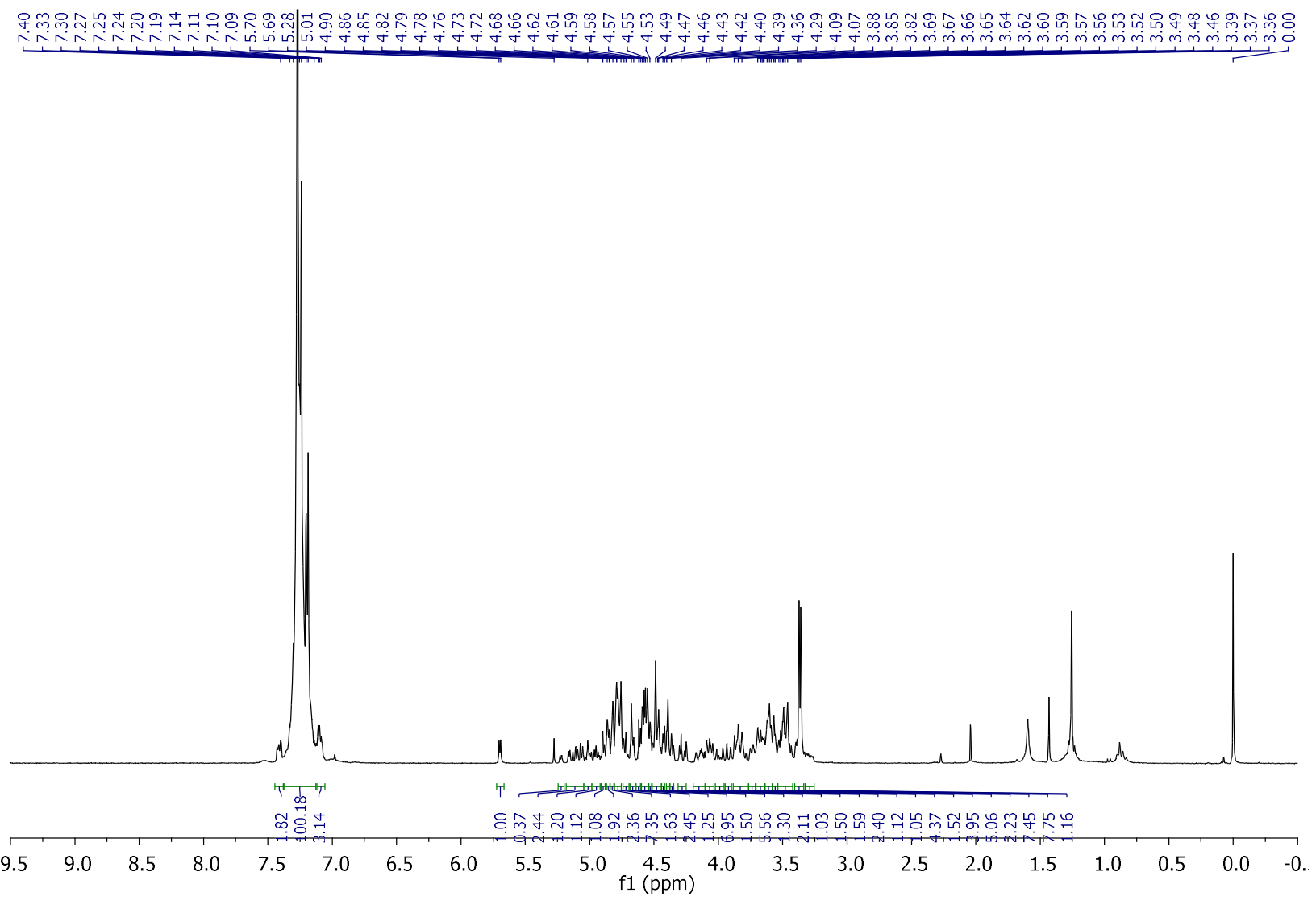




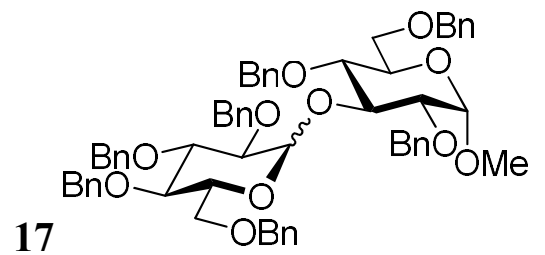

${ }^{1} \mathrm{H} \mathrm{NMR}\left(\mathrm{CDCl}_{3}, 300 \mathrm{MHz}\right)$

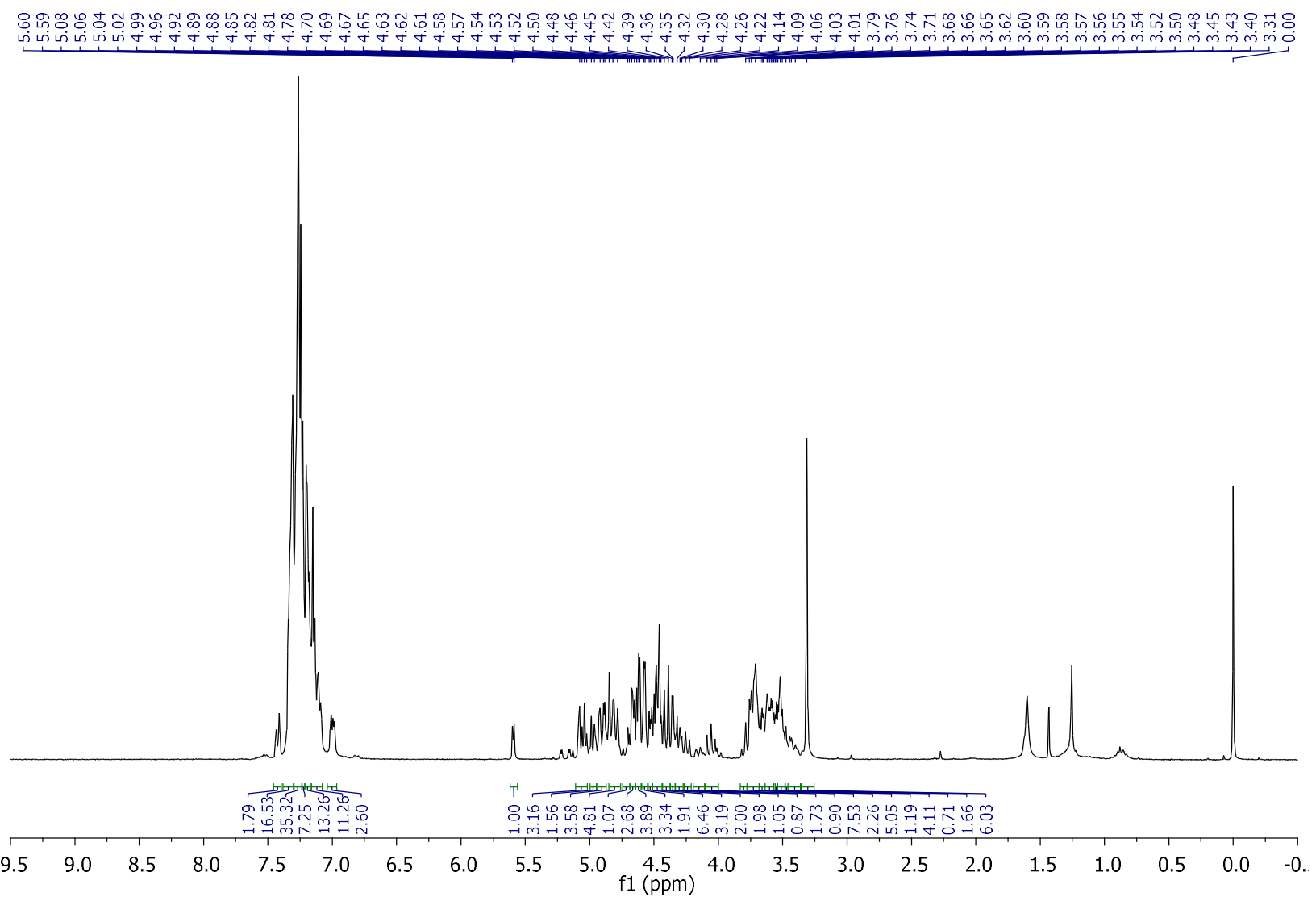




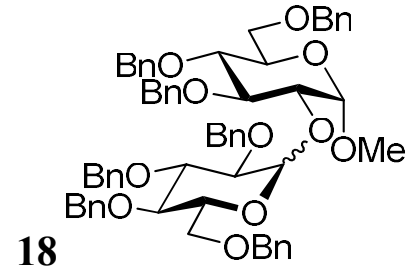

${ }^{1} \mathrm{H} \mathrm{NMR}\left(\mathrm{CDCl}_{3}, 300 \mathrm{MHz}\right)$

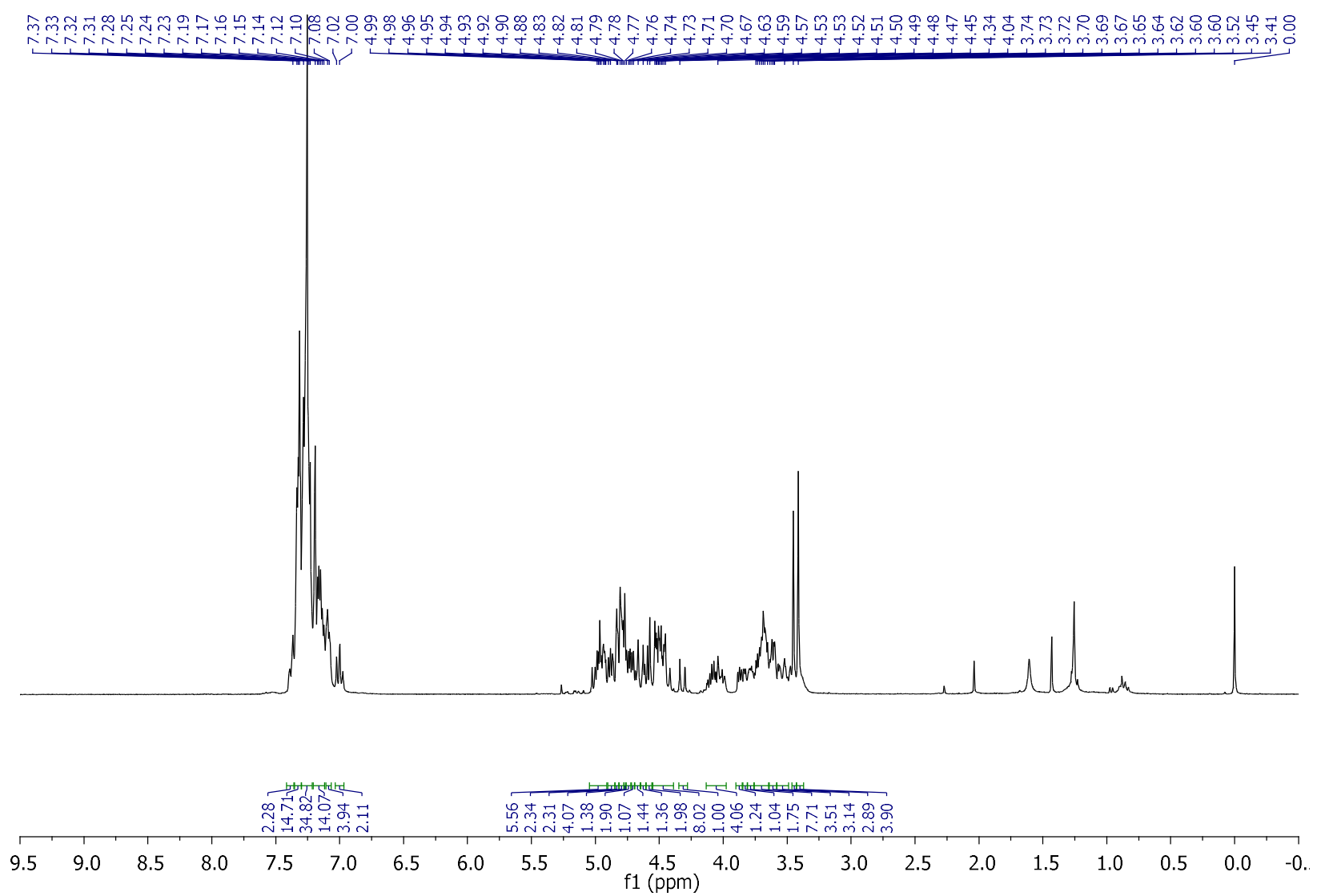




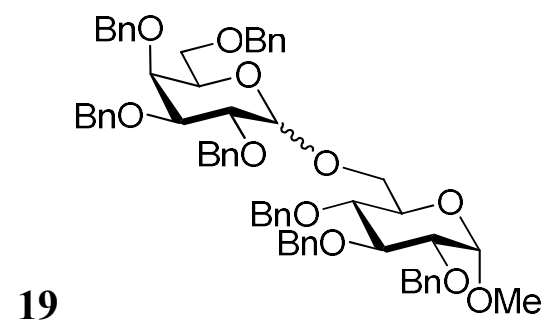

${ }^{1} \mathrm{H} \mathrm{NMR}\left(\mathrm{CDCl}_{3}, 300 \mathrm{MHz}\right)$

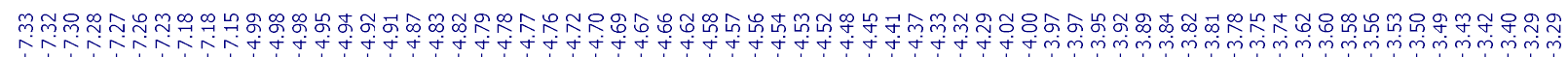

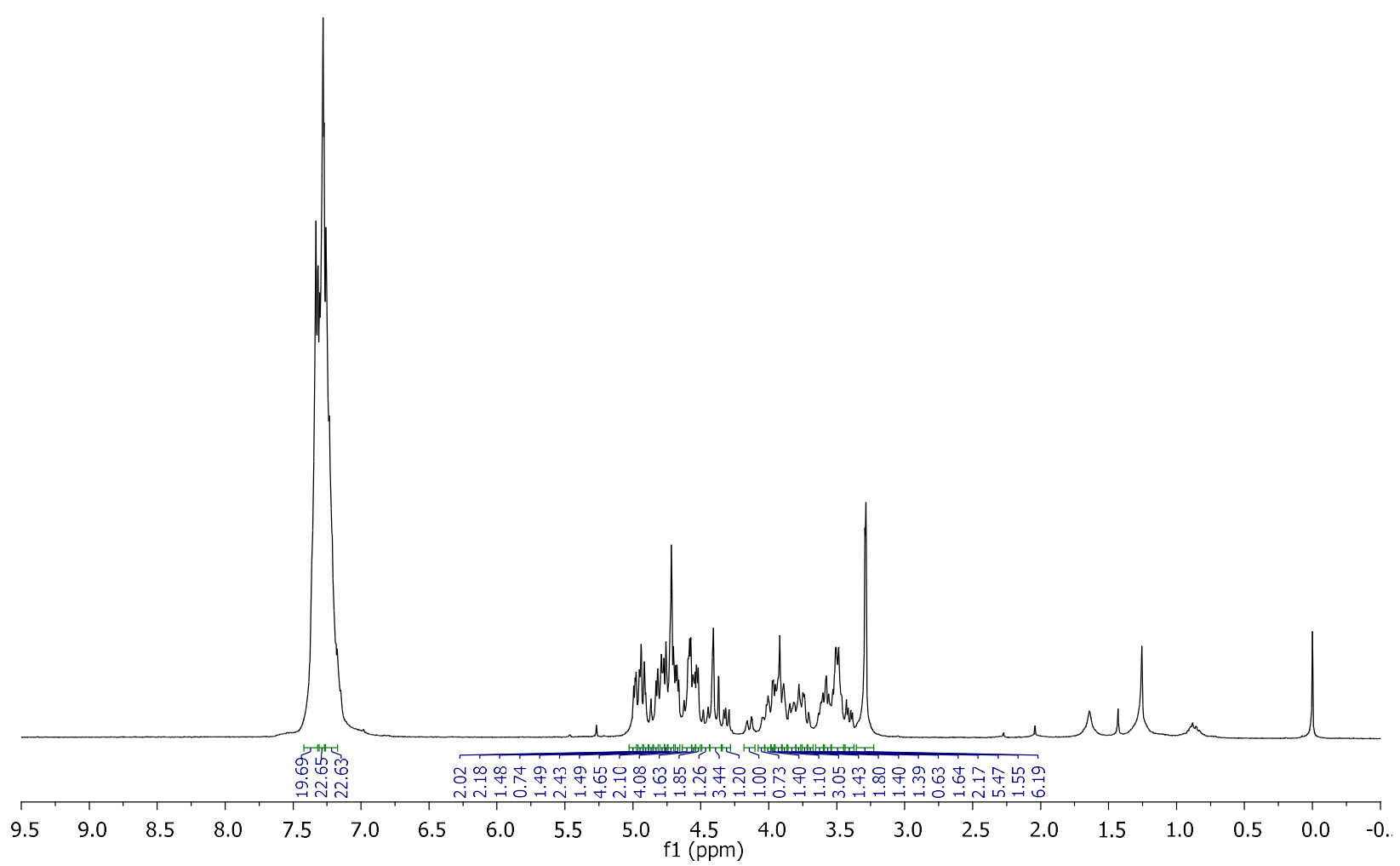




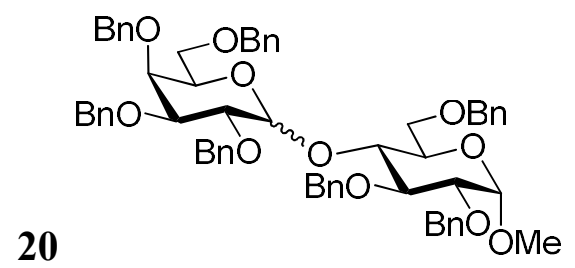

${ }^{1} \mathrm{H} \mathrm{NMR}\left(\mathrm{CDCl}_{3}, 300 \mathrm{MHz}\right)$

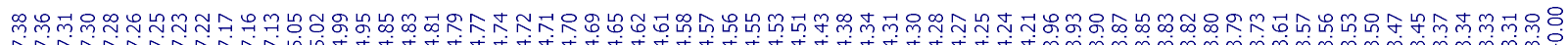
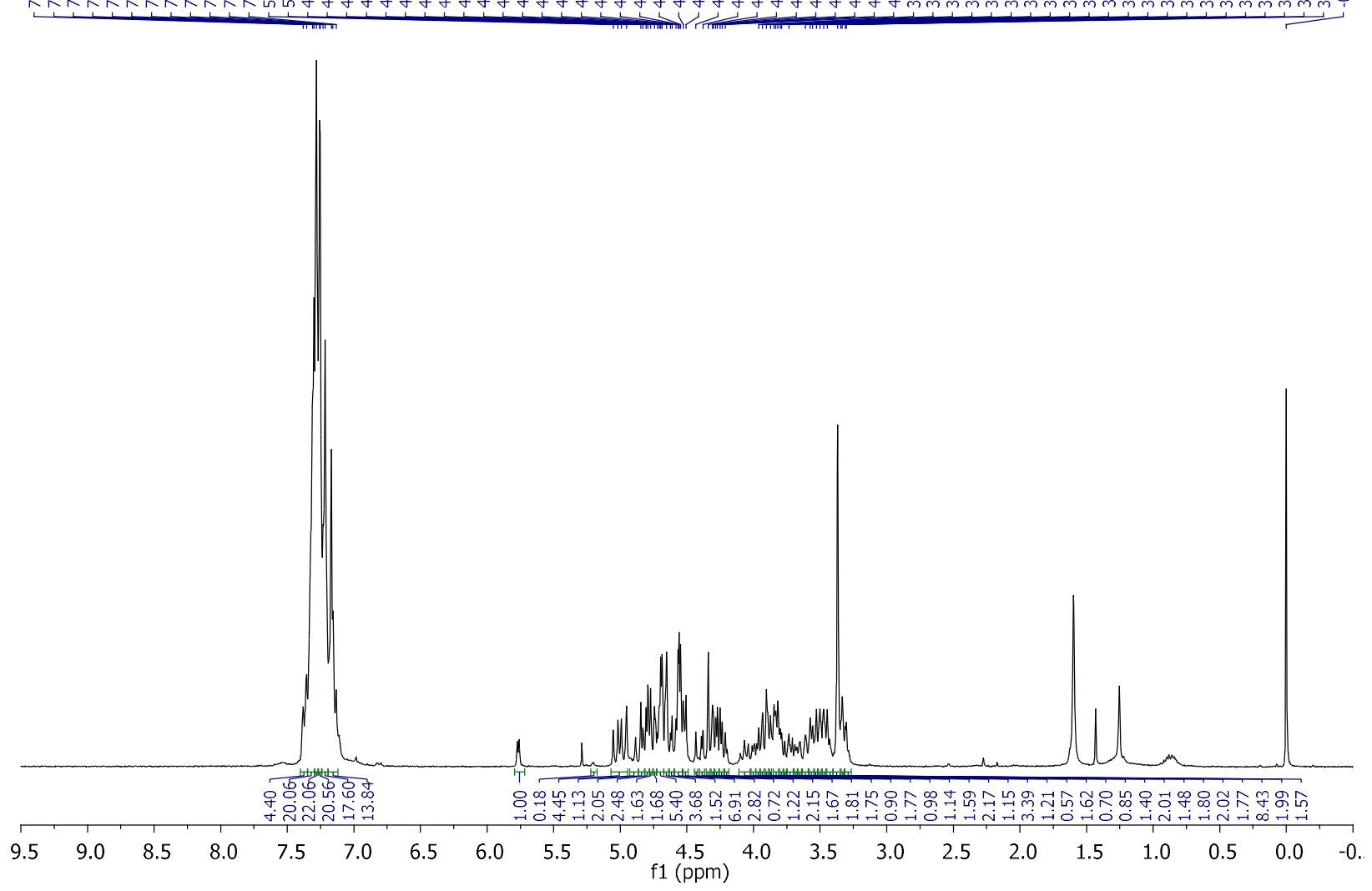


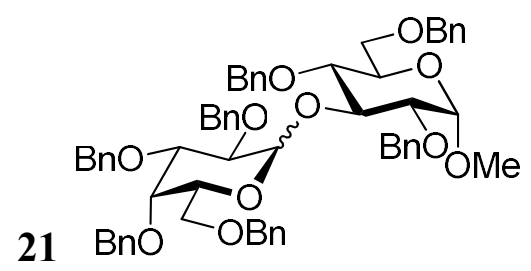

${ }^{1} \mathrm{H} \mathrm{NMR}\left(\mathrm{CDCl}_{3}, 300 \mathrm{MHz}\right)$

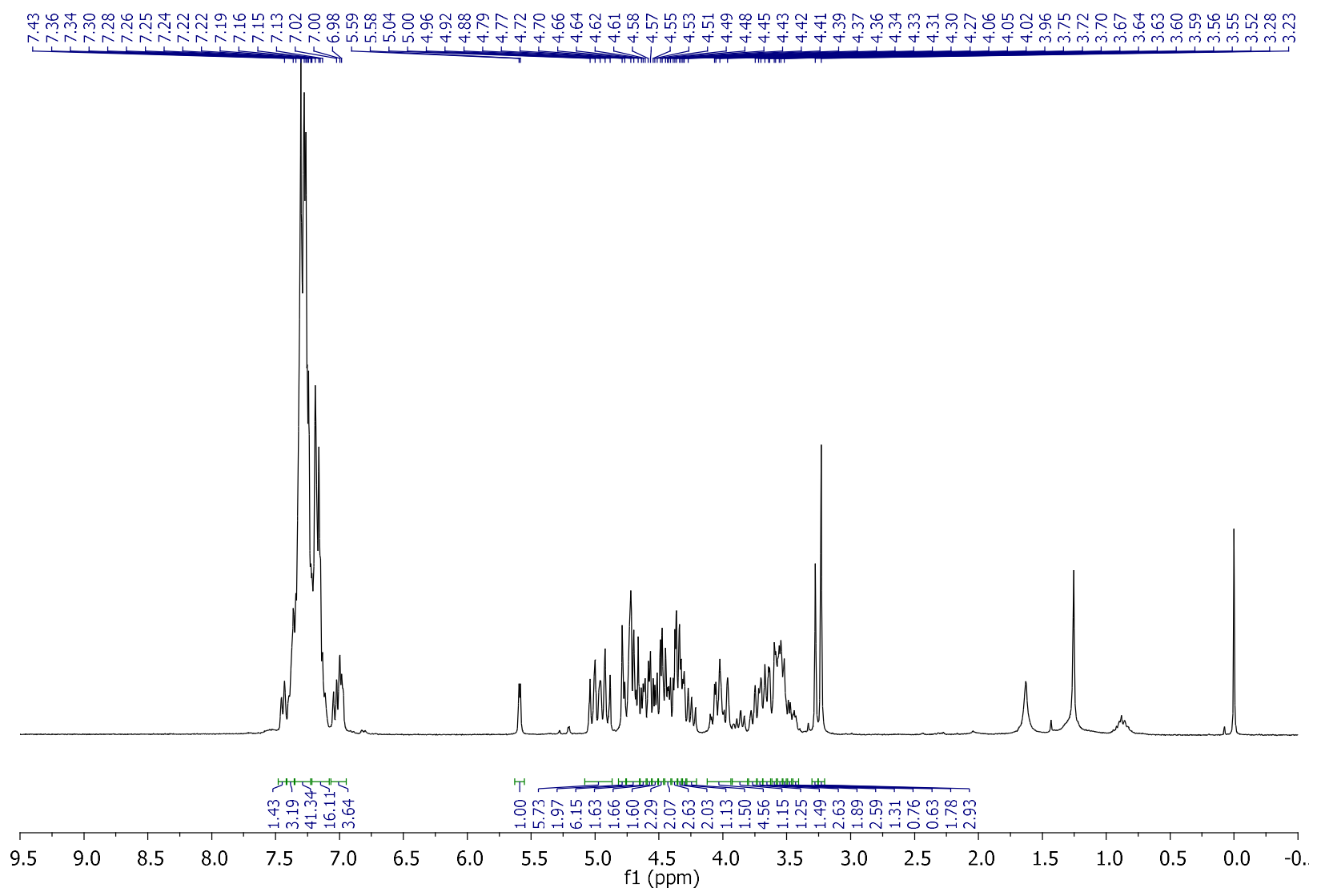




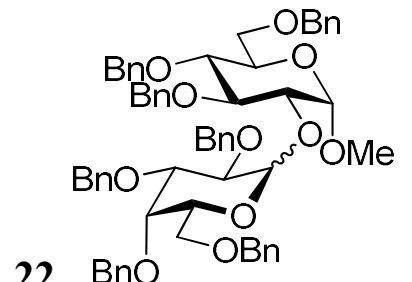

${ }^{1} \mathrm{H} \mathrm{NMR}\left(\mathrm{CDCl}_{3}, 300 \mathrm{MHz}\right)$

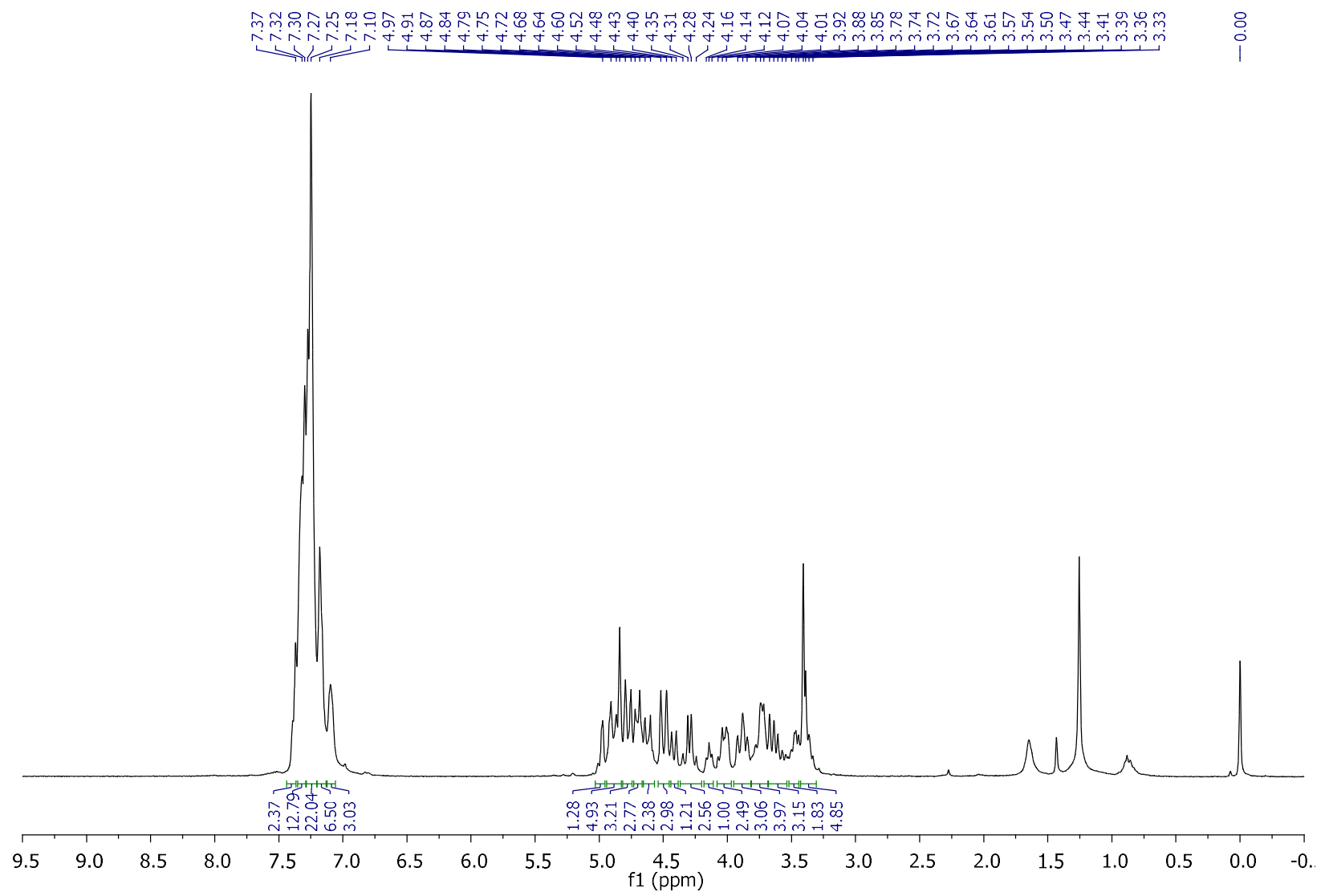




\section{$\underline{X-R a y ~ c r y s t a l ~ s t r u c t u r e ~ d e t e r m i n a t i o n ~ f o r ~ c o m p o u n d ~} 2$}

\section{Table S1. Crystal data and structure refinement for 2 (d1119-180K).}

\begin{tabular}{|c|c|c|}
\hline Identification code & \multicolumn{2}{|c|}{ avd1119-180K/x8/GS2P54 } \\
\hline Empirical formula & \multicolumn{2}{|l|}{$\mathrm{C}_{42} \mathrm{H}_{33} \mathrm{~N} \mathrm{O}_{9} \mathrm{~S}$} \\
\hline Formula weight & \multicolumn{2}{|l|}{727.75} \\
\hline Temperature & \multicolumn{2}{|l|}{$180(2) \mathrm{K}$} \\
\hline Wavelength & \multicolumn{2}{|l|}{$0.71073 \AA$} \\
\hline Crystal system & \multicolumn{2}{|l|}{ Monoclinic } \\
\hline Space group & \multicolumn{2}{|l|}{$\mathrm{P} 21$} \\
\hline Unit cell dimensions & $\begin{array}{l}\mathrm{a}=5.9632(2) \AA \\
\mathrm{b}=18.9516(9) \AA \\
\mathrm{c}=15.9602(7) \AA\end{array}$ & $\begin{array}{l}\alpha=90^{\circ} . \\
\beta=95.889(2)^{\circ} \\
\gamma=90^{\circ} .\end{array}$ \\
\hline $\begin{array}{l}\text { Volume } \\
\text { Z }\end{array}$ & \multicolumn{2}{|l|}{$1794.18(13) \AA^{3}$} \\
\hline Density (calculated) & \multicolumn{2}{|l|}{$1.347 \mathrm{Mg} / \mathrm{m}^{3}$} \\
\hline $\begin{array}{l}\text { Absorption coefficient } \\
F(000)\end{array}$ & \multicolumn{2}{|l|}{$0.150 \mathrm{~mm}^{-1}$} \\
\hline Crystal size & \multicolumn{2}{|c|}{$0.398 \times 0.281 \times 0.233 \mathrm{~mm}^{3}$} \\
\hline Theta range for data collection & \multicolumn{2}{|c|}{2.149 to $27.530^{\circ}$. } \\
\hline Index ranges & \multicolumn{2}{|c|}{$-7 \leq \mathrm{h} \leq 7,-24 \leq \mathrm{k} \leq 24,-20 \leq \mathrm{l} \leq 20$} \\
\hline Reflections collected & \multicolumn{2}{|l|}{24279} \\
\hline Independent reflections & \multicolumn{2}{|c|}{$8263[\mathrm{R}(\mathrm{int})=0.0407]$} \\
\hline Completeness to theta $=25.242^{\circ}$ & \multicolumn{2}{|c|}{$99.9 \%$} \\
\hline Absorption correction & \multicolumn{2}{|c|}{ Semi-empirical from equivalents } \\
\hline Max. and min. transmission & \multicolumn{2}{|l|}{0.9420 and 0.9063} \\
\hline $\begin{array}{l}\text { Refinement method } \\
\text { Data / restraints / parameters }\end{array}$ & \multicolumn{2}{|c|}{$\begin{array}{l}\text { Full-matrix least-squares on } \mathrm{F}^{2} \\
8263 / 2 / 486\end{array}$} \\
\hline Goodness-of-fit on $\mathrm{F}^{2}$ & \multirow{4}{*}{\multicolumn{2}{|c|}{$\begin{array}{l}1.041 \\
\mathrm{R} 1=0.0412, \mathrm{wR} 2=0.0757 \\
\mathrm{R} 1=0.0659, \mathrm{wR} 2=0.0852 \\
0.02(4)\end{array}$}} \\
\hline Final $\mathrm{R}$ indices $[\mathrm{I}>2 \operatorname{sigma}(\mathrm{I})]$ & & \\
\hline $\mathrm{R}$ indices (all data) & & \\
\hline Absolute structure parameter & & \\
\hline Largest diff. peak and hole & \multicolumn{2}{|c|}{0.146 and -0.179 e. $\AA^{-3}$} \\
\hline
\end{tabular}


Figure S11. Crystal projection view with $\mathbf{5 0 \%}$ probability ellipsoids for compound 2 :

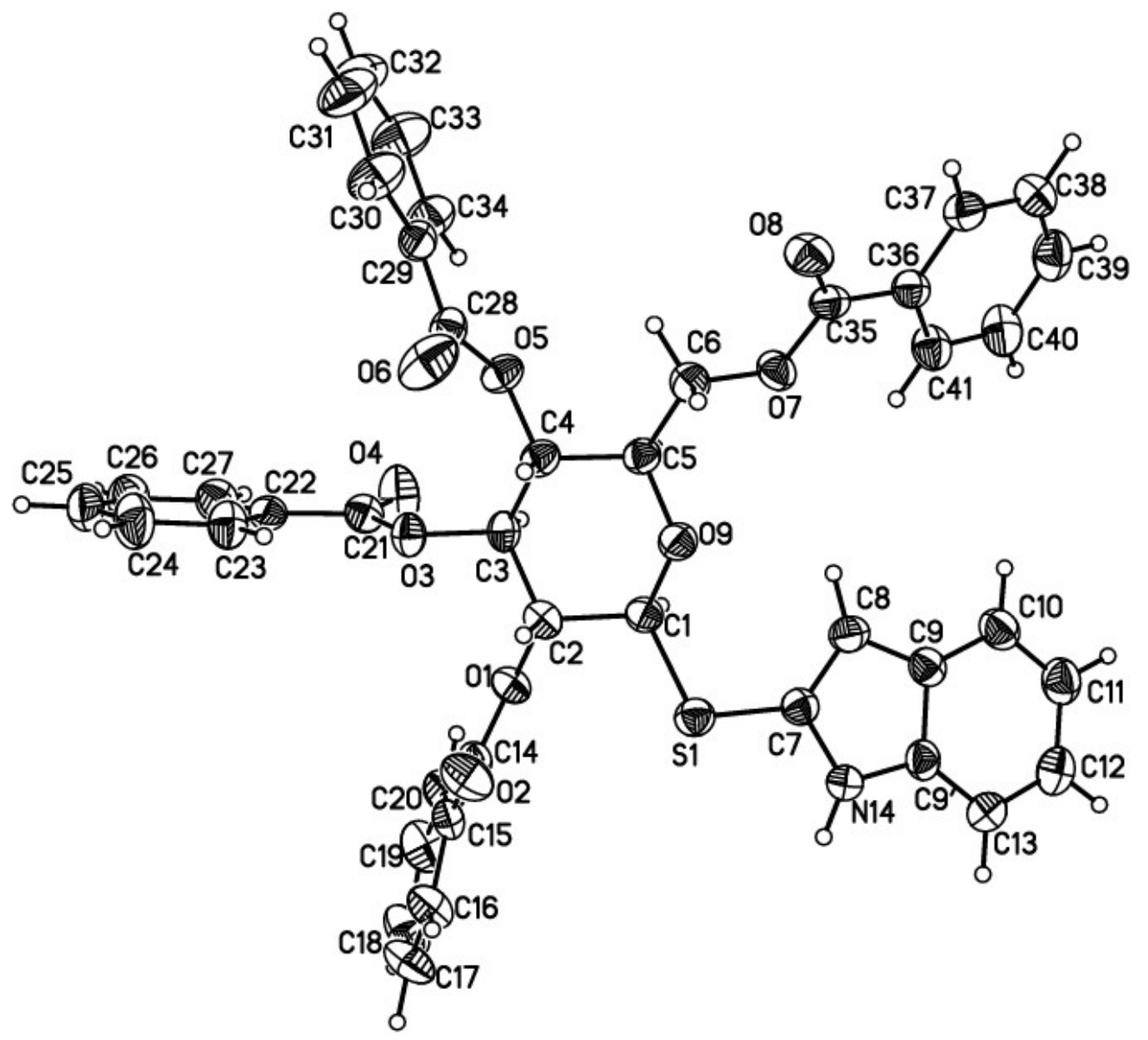




\section{$\underline{\text { X-Ray crystal structure determination for compound } 6}$}

Table S2. Crystal data and structure refinement for 6 (avd15819).

Identification code

Empirical formula

Formula weight

Temperature

Wavelength

Crystal system

Space group

Unit cell dimensions

Volume

Z

Density (calculated)

Absorption coefficient

$\mathrm{F}(000)$

Crystal size

Theta range for data collection

Index ranges

Reflections collected

Independent reflections

Completeness to theta $=67.679^{\circ}$

Absorption correction

Max. and min. transmission

Refinement method

Data / restraints / parameters

Goodness-of-fit on $\mathrm{F}^{2}$

Final $\mathrm{R}$ indices [I $>2 \operatorname{sigma}(\mathrm{I})]$

$\mathrm{R}$ indices (all data)

Absolute structure parameter

Largest diff. peak and hole
d15819/1t/venture/Shrestha

$\mathrm{C}_{42} \mathrm{H}_{41} \mathrm{~N} \mathrm{O}_{5} \mathrm{~S}$

671.82

$100(2) \mathrm{K}$

$1.54178 \AA$

Monoclinic

$\mathrm{P} 21$

$\mathrm{a}=11.9289(4) \AA \quad \alpha=90^{\circ}$.

$\mathrm{b}=8.8193(3) \AA \quad \beta=100.021(2)^{\circ}$.

$\mathrm{c}=16.9246(6) \AA \quad \gamma=90^{\circ}$.

$1753.38(11) \AA^{3}$

2

$1.272 \mathrm{Mg} / \mathrm{m}^{3}$

$1.194 \mathrm{~mm}^{-1}$

712

$0.198 \times 0.063 \times 0.032 \mathrm{~mm}^{3}$

2.651 to $74.608^{\circ}$.

$-14 \leq \mathrm{h} \leq 14,-10 \leq \mathrm{k} \leq 10,-21 \leq \mathrm{l} \leq 20$

25115

$7005[\mathrm{R}($ int $)=0.051]$

$99.8 \%$

Semi-empirical from equivalents

0.8645 and 0.7030

Full-matrix least-squares on $\mathrm{F}^{2}$

7005 / 4 / 452

1.059

$\mathrm{R} 1=0.0393, \mathrm{wR} 2=0.0872$

$\mathrm{R} 1=0.0433, \mathrm{wR} 2=0.0896$

$-0.011(11)$

0.344 and -0.171 e. $\AA^{-3}$ 
Figure S12. Crystal projection view of compound 6 with 50\% probability ellipsoids disorder component omitted for clarity:

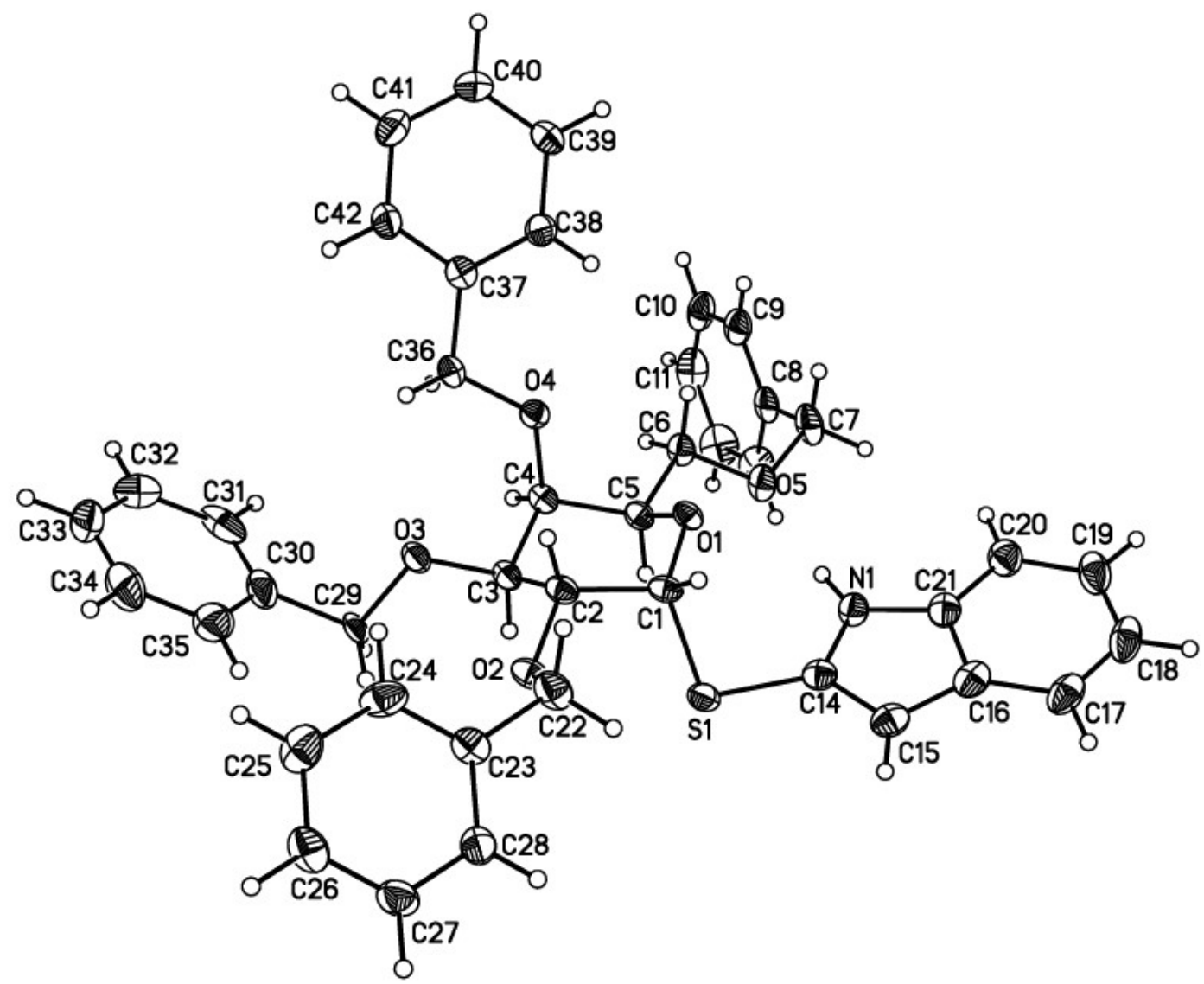

